\title{
A computer experiment on diffusion in the Lorentz gas
}

C. Bruin

\section{Delft University Press}


A computer experiment on diffusion in the Lorentz gas

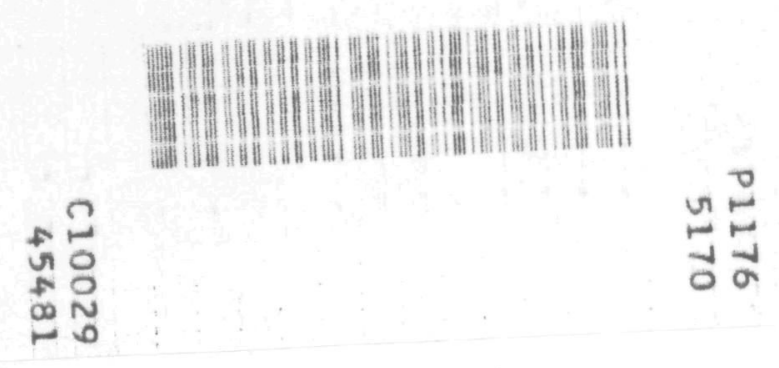

BIBLIOTHEEK TU Delft 


\section{A computer experiment on diffusion in the Lorentz gas}

Proefschrift ter verkrijging

van de graad van doctor in de

technische wetenschappen

aan de Technische Hogeschool Delft, op gezag van de rector magnificus

prof. ir. L. Huisman,

voor een commissie aangewezen

door het college van dekanen

te verdedigen op

woensdag 1 maart 1978

te 16.00 uur door

Cornelis Bruin

natuurkundig ingenieur

geboren te 's-Gravenhage

Delft University Press / 1978

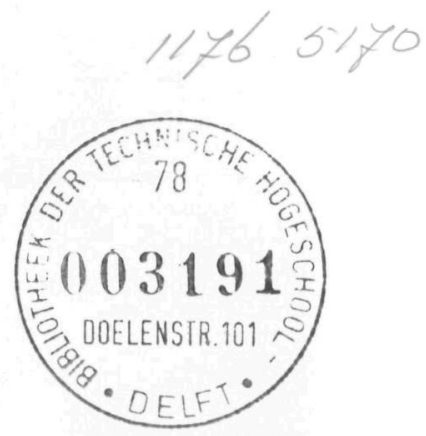


Dit proefschrift is goedgekeurd door de promotor prof. dr. J. M. J. van Leeuwen 
Aan Tine

Ralph

Niki

Nils 
CONTENTS

1 INTRODUCTION 9

1.1 Computer experiments 9

1.2 Diffusion in the Lorentz gas 11

$\begin{array}{llr}1.3 \text { Survey } & 12\end{array}$

References 14

2 THEORY $\quad 17$

$\begin{array}{lr}2.1 & 17\end{array}$

2.2 Density expansion 20

2.2.1 Outzine of the expansion method 20

2.2.2 First few expansion coefficients 23

2.2.3 Higher-order regular contributions in two dimensions 27

2.2.4 Higher-order singular contributions in two dimensions 30

2.2.5 Velocity autocorrelation function 32

2.3 Free-time distribution 33

2.4 Finite-size effects 39

$\begin{array}{ll}\text { References } & 41\end{array}$

3 METHOD OF COMPUTATION $\quad 43$

3.1 Monte Carlo method 43

3.2 Molecular-dynamics method 45

$\begin{array}{lll}3.3 & \text { Correlation functions } & 48\end{array}$

3.4 Diffusion coefficients 50

3.5 Mean free time and distribution of free times 51

References $\quad 53$ 
4

COMPUTATIONAL RESULTS

4.1 Two-dimensional Lorentz gas

4.2 Three-dimensional Lorentz gas

4.3 Results on the $\mathrm{N}$-dependence

APPENDIX The joint distribution of the first and second free time for the two-dimensional case

SUMMARY

SAMENVATTING 


\section{INTRODUCTION}

\subsection{Computer experiments}

In the last two decades the use of electronic computers in statistical mechanics has developed from being just a tool for computing approximate values of configuration integrals to an alternative way of doing experiments, or at least to a way of getting quasi-experimental results for theoretical models.

Some noteworthy points in this development will be mentioned here. In 1953 Metropolis et al.1.1) computed values for the configuration integral of a two-dimensional system of 224 hard disks by means of the Monte Carlo method. They used a modification of the crude Monte Carlo method, generating a Markov chain of configurations with transition probabilities giving rise to the canonical distribution function for the configurations in the limit of infinitely long Markov chains. The Monte Carlo method gives only equilibrium properties, but the succession of configurations in a Markov chain can be thought of as a quasi-kinetic behaviour of the system. This concept is used e.g. in the kinetic Ising model 1.2).

Soon afterwards (1956) Alder and Wainwright ${ }^{1.3)}$ introduced the molecular-dynamics method by solving numerically the equations of motion of systems with a hard-core interaction. They obtained in this way static quantities (related to the equation of state) as well as timedependent ones as the velocity autocorrelation function.

A breakthrough to more realistic systems was made in 1964 by Rahman. He solved the equations of motion, written as difference equations, of a system of 864 particles with a Lennard-Jones interaction chosen to simulate liquid argon ${ }^{1.4}$ ). He was the first to stress the relevance of these computations for quasi-elastic neutron scattering. He computed in this spirit apart from the velocity autocorrelation function also 
the density-density correlation function in space and time. Later on, not only the self-diffusion coefficient but also bulk and shear viscosities and the heat conductivity that are much harder to calculate for such a system have been determined. ${ }^{1.5)}$ This method, suitable for systems with any interaction potential given in an analytic or tabulated form, is presently being applied for the simulation of such complicated systems like water ${ }^{1.6)}$, molten salts ${ }^{1.7)}$ and plasmas. ${ }^{1.8)}$

As a final example, the conceptually important result is mentioned of Alder and Wainwright ${ }^{1.9)}$ in 1970 , i.e. the discovery of the longtime tails of the velocity autocorrelation function.

This thesis is concerned with a molecular-dynamics study of a problem which is also mostly of a conceptual. nature. In the study of the density dependence of transport coefficients Dorfman and Cohen ${ }^{1.10}$ ) discovered that a power-series expansion of the transport coefficients is most likely to be impossible. Their argument is based on a phase space estimate of the integrals yielding the higher-order density corrections to the low-density (Boltzmann) values of the transport quantities. As the restriction imposed by the (irreducible) N-body dynamics on the phase space is not sufficient to guarantee the convergence of the integrals over the pertinent collision sequences, they concluded that the density series proposed so-far was divergent in sufficiently high order (depending on the dimensionality of the system).

We will show that the so-called Lorentz gas is an ideal moleculardynamics testing ground for this issue. The Lorentz gas is a random assembly of spherical scatterers through which one spherical particle moves. The fixed scatterers are chosen to be point-particles and cannot move. Another type of Lorentz gas, the wind-tree model due to Ehrenfest, has been studied in another context by wood and Lado ${ }^{1.11)}$ in this model a point-particle is scattered by fixed parallel squares.

The phase space of the Lorentz gas is $(\mathrm{N}+2)$ d dimensional, $d$ being the dimensionality of the system and $N$ the number of scatterers. Since the absolute value of the momentum of the moving particle is a constant of motion, trajectories always remain on the same sphere in 
momentum space. This makes integrals over momentum space trivial. The positions of the scatterers are not affected by the time evolution of the system, and therefore a Monte Carlo procedure is needed to sample their Nd-dimensional configuration space. The trajectory of the moving particle in a given configuration of the scatterers can be computed by means of molecular dynamics in a simplified version with respect to Alder and Wainwright ${ }^{1.12)}$; there being only one moving particle with constant velocity. In this way phase space can be sampled properly if enough computing time is available and if thereare no closed pockets in the generated configurations of scatterers. Such closed pockets, which are disconnected regions in the configuration space of the moving particle, can be dealt with by a Monte Carlo procedure for the generation of initial positions of the moving particle.

The computations presented here are an example of obtaining quasiexperimental results for a theoretical model that has no clear-cut analogue in nature.

\subsection{Diffusion in the Lorentz gas}

Transport coefficients can be calculated from equilibrium averages of time correlation functions. These functions provide also a particularly suitable starting point for the investigation of the density dependence of transport coefficients. Zwanzig ${ }^{1.13}$ ) designed a simple scheme for computing the coefficients of a power-series expansion in the density for transport coefficients. However, as mentioned before, actual calculations lead to divergencies in certain expansion coefficients. Proper resummation gave rise to terms logarithmic in the density 1.14-1.17).

Although these theoretical predictions date from several years ago, there is as yet no experimental confirmation for the existence of logarithmic terms. The reason is that for real systems the coefficients of these terms are unknown and, probably, small ${ }^{1.18)}$. This smallness is supported by the result of the explicit calculation ${ }^{1.16,1.17)}$ for the three-dimensional Lorentz gas of the coefficient of the first 
logarithmic term in the density expansion for the diffusion coefficient.

For the two-dimensional Lorentz gas the coefficient of the first logarithmic term turns out to be comparatively large ${ }^{1.17)}$. The main objective of the present study is to find confirmation for the existence of this term from combined Monte carlo and molecular-dynamics calculations (for which the model is very well suited).

Another reason for studying the Lorentz gas is provided by Ernst and Weyland 19). They derived an expression for the earlier mentioned long-time behaviour of the velocity autocorrelation function for a d-dimensional Lorentz gas at low densities. In systems with complete hydrodynamics like the hard-sphere gas the expression has a positive sign and contains an inverse power of time: $t^{-d / 2}$; see refs. ${ }^{1.9}, 1.20$ ), and ref ${ }^{1.21)}$. For the Lorentz gas, where diffusion is the only hydrodynamic mode, the expression has a negative sign and a $t^{-\frac{1}{2} d-1}$ behaviour. Therefore the diffusion coefficient, which is the time integral over the velocity autocorrelation function, remains finite also in two dimensions. This asymptotic behaviour of the velocity autocorrelation function is a very small effect.

1.3. Survey

In chapter 2 theoretical considerations on the Lorentz gas are presented. In section 2.1 the Lorentz gas is defined, together with the most important quantities. In the first part of section 2.2 an outline of the expansion method is given, whereas the second part gives a summary of the calculations of van Leeuwen and Weyland for the expansion coefficients up to the first logarithmic ones both for $d=2$ and $d=3$. In sub-section 2.2.3 the diagrams giving a regular contribution to the same order in the density as the first logarithmic term are listed together with their contributions for the two-dimensional case. The numerical computations are discussed briefly. In sub-section 2.2.4 the diagrams contributing to the squared logarithmic term, one order higher in the density, are listed together with their contributions, which could be obtained analytically. In sub-section 2.2.5 the Boltzmann expressions 
are given for the velocity autocorrelation function as well as the expressions for asymtotic long times found by Ernst and Weyland.

In section 2.3 expressions are derived for the free-time distribution and the mean free time. These quantities have been chosen as a test on the randomness of the scatterers and they serve the purpose rather well. The distribution of first free times is purely exponential but the freetime distribution (including second, third, etc. free times as well) must be handled with care. It turns out to be essential in what way one takes averages over the results of different configurations. In the appendix an explicit calculation is given, for the two-dimensional case, of the joint distribution of the first and second free time, leading to a nonexponential behaviour for the distribution of second free times. The chapter closes with some considerations on finite-size effects.

The method of computation is explained in chapter 3, starting with the crude Monte Carlo method for generating different configurations of scatterers. In section 3.2 the computation of trajectories by means of molecular dynamics is described. In the next section different ways of obtaining the velocity autocorrelation function and the mean square displacement are discussed. The computational method for obtaining the diffusion coefficient from these functions is given in section 3.4. The computation of the mean free time and distribution of free times is dealt with in section 3.5, where the different ways of taking averages are emphasized.

In chapter 4 the computer results are presented on the mean free time, the distribution of free times, the velocity autocorrelation function, the mean-square displacement and the diffusion coefficient.

In chapter 5 the conclusions are summarized. 
References

1.1) Metropolis, N., et al., J. Chem. Phys. 21 (1953) 1087

1.2) Compagner, A., J. Phys. Soc. Japan Suppl. 26 (1969) 229 Stoll, E., Binder, K. and Schneider, T., Phys. Rev. B 8 (1973) 3266

1.3) Alder, B.J. and Wainwright, T.E., (1956) in Internat. Symp. on Stat. Mech. Theory of Transport Processes. Ed. I. Progogine, Interscience, Brussels 1958

1.4) Rahman, A., Phys. Rev. 136 (1964) A405

1.5) Bruin, C., Phys. Letters 28A (1969) 777

1.6) Rahman, A. and Stillinger, F.H., J. Chem. Phys. 55 (1971) 3336

1.7) Hansen, J.P. and McDonald, I.R., J. Phys. C $\underline{7}$ (1974) L384

1.8) Hockney, R.W., Goel, S.P. and Eastwood, J.W., Chem.Phys. Letters 21 (1973) 589

1.9. Alder, B.J. and Wainwright, T.E., Phys. Rev. A1 (1970) 18

1.10) Dorfman, J.R. and Cohen, E.G.D., Phys. Letters 16 (1965) 124

1.11) Wood, W.W. and Lado, F., J. Comp. Phys. 7 (1971) 528

1.12) Alder, B.J. and Wainwright, T.E., J. Chem. Phys. 31 (1959) 459

1.13) Zwanzig, R., Phys. Rev. 129 (1963) 486

1.14) Sengers, J.V., Phys. Rev. Letters 15 (1965) 515

1.15) Haines, L.K., Dorfman, J.R. and Ernst, M.H., Phys. Rev. 144 (1966) 207

1.16) Van Leeuwen, J.M.J. and Weyland, A., Physica 36 (1967) 457

1.17) Weyland, A. and Van Leeuwen, J.M.J., Physica 38 (1968) 35

1.18) Kestin, J., Paykoq, E. and Sengers, J.V., Physica 54 (1971) 1 
1.19) Ernst, M.H. and Weyland, A., Phys. Letters 34A (1971) 39

1.20) Ernst, M.H., Hauge, E.H. and Van Leeuwen, J.M.J., Phys. Re'v. Letters $\underline{25}$ (1970) 1254

1.21) Dorfman, J.R. and Cohen, E.G.D., Phys. Rev, Letters 25 (1970) 1257 
2. THEORY

\subsection{Definition of the model}

The essential feature of a Lorentz gas is that only one particle moves in an assembly of static particles. In the classical case one has for the Hamilton function

$$
H\left(x, R^{N}\right)=\frac{p^{2}}{2 m}+\sum_{j=1}^{N} V_{d y}\left(\vec{q}-\vec{R}_{j}\right)+\frac{1}{2 !} i \sum_{j} V_{s t}\left(\vec{R}_{i}-\vec{R}_{j}\right),
$$

where $x$ stands for $(\vec{p}, \vec{q}), \vec{q}$ is the position and $\vec{p}=m \vec{v}$ the momentum of the moving particle, $m$ being its mass and $\vec{v}$ its velocity. The coordinates of the $N$ static particles are denoted by $R^{N}=\left(\vec{R}_{1}, \ldots, \vec{R}_{N}\right)$. For the dynamical part of the interaction $V_{d y}\left(\vec{q}-\vec{R}_{j}\right)$, operating between the moving particle and scatterer $j$, we take a spherically symmetric hardcore potential

$$
v_{d y}\left(\vec{q}-\vec{R}_{j}\right)=\begin{aligned}
& \infty \text { for }\left|\vec{q}-\vec{R}_{j}\right| \leqslant \sigma, \\
& 0 \text { for }\left|\vec{q}-\vec{R}_{j}\right|>\sigma .
\end{aligned}
$$

Similarly, for the interaction $v_{s t}\left(\vec{R}_{i}-\vec{R}_{j}\right)$ between two scatterers we take

$$
\begin{array}{ll}
V_{s t}\left(\vec{R}_{i}-\vec{R}_{j}\right)= & \text { for }\left|\vec{R}_{i}-\vec{R}_{j}\right| \leqslant \sigma_{s}, \\
0 & \text { for }\left|\vec{R}_{i}-\vec{R}_{j}\right|>\sigma_{s} .
\end{array}
$$

Furthermore we will assume the scatterers to be point-particles, $\sigma_{\mathrm{S}}=0$, so the third term in $(2.1)$ is zero.

With these specifications our model is a system of $\mathrm{N}$ point-scatterers 
between which a single sphere of radius $\sigma$ is moving. Alternatively the model can be looked at as being a system of $\mathrm{N}$ possibly overlapping scatterers of radius $\sigma$ with one moving point-particle. The system is enclosed in a d-dimensional box of volume $\Omega$. Instead of the density of scatterers $\mathrm{n}=\mathrm{N} / \Omega$, the reduced density $\mathrm{n}^{*}=\mathrm{no}^{\mathrm{d}}$ will be used, which is dimensionless. Strictly speaking, it is necessary for the analysis below to take the thermodynamic limit $(\mathrm{N} \rightarrow \infty$ and $\Omega \rightarrow \infty$, keeping $\mathrm{n}$ fixed).

The diffusion coefficient of the moving particle is the quantity of most interest in this study. The relation between the diffusion coefficient $D_{T}$ at a certain temperature $T$ and the velocity autocorrelation function reads

$$
\left.D_{T}=\frac{1}{d} \int_{0}^{\infty} d t<\vec{v}(0) \cdot \vec{v}(t)\right\rangle_{T},
$$

where $\vec{v}(t)$ denotes the velocity of the moving particle at time $t$ and the average $\langle\ldots\rangle_{T}$ is a canonical average over all distributions of the scatterers and of the moving particle (with temperature $\mathrm{T}$ ). One can write (2.4) in more detail,

$$
D_{T}=\frac{1}{d} \int_{0}^{\infty} d t \int d x \int d R^{N} \rho\left(x, R^{N}\right) \vec{v} \cdot\left\{\exp t L\left(x, R^{N}\right)\right\} \vec{v}
$$

making use of the Liouville operator $L\left(x, R^{N}\right)$ defined by

$$
\left\{\exp t L\left(x, R^{N}\right)\right\} x=x(t), \quad x(0)=x,
$$

and the canonical distribution function given by

$$
\rho\left(x, R^{N}\right)=\left\{\exp -\beta H\left(x, R^{N}\right)\right\} / \int d x \int d R^{N} \exp -\beta H\left(x, R^{N}\right)
$$

with $\beta=1 / \mathrm{kT}, \mathrm{k}$ being Boltzmann's constant.

The absolute value $\mathrm{v}$ of the velocity of the moving particle is a constant of motion. Therefore the average over the Maxwell-Boltzmann distribution $\varphi_{T}(\vec{v})$, needed to obtain results at a given temperature $T$, does not interfere with the spatial averages. Denoting by $\langle\ldots\rangle$ the 
average over the spatial coordinates only, we find for the average at fixed temperature $\mathrm{T}$ :

$$
\langle\vec{v}(0) \cdot \vec{v}(t)\rangle_{T}=\int d \vec{v} \varphi_{T}(\vec{v})\langle\vec{v}(0) \cdot \vec{v}(t)\rangle,
$$

where in the average on the right-hand side the absolute value $\mathrm{v}$ of the velocity of the moving particle is kept fixed. Apart from a trivial factor $v^{2}$, the velocity dependence of $\langle\vec{v}(0) \cdot \vec{v}(t)\rangle$ appears only in the form of a scale factor in the time dependence. Thus we may write

$$
\vec{v}(0) \cdot \vec{v}(t)\rangle=v^{2} \psi(v t / \sigma)
$$

where a factor $\sigma$ is included to define a reduced time

$$
t^{*}=v t / \sigma .
$$

Inserting (2.8) and (2.9) into (2.4) and using $t^{*}$ as an integration variable one finds

$$
D_{T}=\frac{\Gamma\left(\frac{1}{2} d+\frac{1}{2}\right)}{\Gamma\left(\frac{1}{2} d+1\right)}\left[\frac{k T}{2 m}\right]^{\frac{1}{2}} \sigma \int_{0}^{\infty} d t^{*} \psi\left(t^{*}\right) .
$$

For our purpose it is more convenient to use a diffusion coefficient D for a fixed velocity $v$, for which one easily obtains

$$
D=\frac{1}{d} \int_{0}^{\infty} d t\langle\vec{v}(0) \cdot \vec{v}(t)\rangle=\frac{v \sigma}{d} \int_{0}^{\infty} d t^{*} \psi\left(t^{*}\right)
$$

Another useful scaling quantity is the Boltzmann mean free time $\tau$, which will be discussed in detail in section 2.3. Since the scatterers are point-particles the collision rate $\Gamma$, the number of collisions per unit time, is easily calculated. One obtains for its inverse , the reduced mean free time $\tau^{*}=v \tau / \sigma$, the expression

$$
\tau^{*}=1 / a_{d} n^{*},
$$


where $a_{d}$ is the reduced cross section, given by $a_{1}=1, a_{2}=2$ and $a_{3}=\pi$. Defining a reduced time $s=t^{*} / \tau^{*}$ and subsequently a reduced velocity autocorrelation function $\phi(s)=\psi\left(t^{*}\right)$, we find from (2.12) the reduced diffusion coefficient

$$
\mathrm{D} / \mathrm{v} \sigma=\frac{\tau^{*}}{\mathrm{~d}} \int_{0}^{\infty} \mathrm{ds} \phi(\mathrm{s})
$$

From a numerical point of view it may be convenient to calculate alternatively the diffusion coefficient from the slope of the mean square displacement at long times:

$$
D / v \sigma=\lim _{s \rightarrow \infty} \frac{\tau^{*}}{2 \mathrm{~d}} \frac{\mathrm{d} \Delta(\mathrm{s})}{\mathrm{ds}},
$$

where

$$
\Delta(s)=\left\langle[\vec{q}(s)-\vec{q}(0)]^{2}\right\rangle / \sigma^{2},
$$

$\vec{q}(s)$ being the position of the moving particle at time $s$.

\subsection{Density expansion}

\subsubsection{Outline of the expansion method}

The scheme for the density expansion of the diffusion coefficient (or rather of its inverse) has been given in detail by Van Leeuwen and Weyland ${ }^{2.1)}$ for the two- and three-dimensional Lorentz gas of spherical particles. It can be summarized as follows. First one performs an Ursell expansion on the time-evolution operator $\exp t L\left(x, R^{N}\right)$ in eq. (2.5). This already gives a density expansion for the velocity autocorrelation function

$$
\psi\left(t^{*}\right)=1+\sum_{l=1}^{\infty} n^{* l} \tilde{\beta}_{\ell}\left(t^{*}\right),
$$


where the coefficient $\tilde{\beta}_{\ell}\left(t^{*}\right)$ denotes the contribution of the $\ell$-particle Ursell operator. Unfortunately, the coefficient $\tilde{\beta}_{\ell}\left(t^{*}\right)$ increases with time as $t^{*}$. Therefore the time integral over these terms diverges for $t^{*} \rightarrow \infty$. This is not surprising, since a density expansion for the diffusion coefficient itself does not exist, the diffusion coefficient becoming larger and larger the more dilute the system of scatterers is. Therefore one should rather aim at a density expansion for the inverse diffusion coefficient.

For that purpose it is convenient to study the Laplace transform $\hat{\psi}\left(z^{*}\right)$ of the velocity autocorrelation function $\psi\left(t^{*}\right)$ :

$$
\tilde{\psi}\left(z^{*}\right)=\int_{0}^{\infty} e^{-z^{*} t^{*}} \psi\left(t^{*}\right) d t^{*} .
$$

According to $(2.12) \mathrm{D} / \mathrm{v} \sigma$ is related to $\hat{\psi}\left(z^{*}\right)$ as

$$
\mathrm{D} / \mathrm{v} \sigma=\lim _{\mathrm{z}^{*} \rightarrow 0} \frac{1}{\mathrm{~d}} \hat{\psi}\left(z^{*}\right),
$$

where we have deliberately used the limit $z^{*} \rightarrow 0$ notation in order to deal with anticipated long-time divergencies, which demonstrate themselves in singular small $z^{*}$ behaviour. From (2.5) and (2.19) the following formal expression for $\bar{\psi}\left(\mathrm{z}^{*}\right)$ can be obtained:

$$
\hat{\psi}\left(z^{*}\right)=v^{-2} \int d \vec{q} \int d R^{N} \rho\left(\vec{q}, R^{N}\right) \vec{v} \cdot\left[z-L\left(x, R^{N}\right)\right]^{-1} \vec{v},
$$

where $\rho\left(\vec{q}, R^{N}\right)$ is the microcanonical distribution function, which is suitable for obtaining spatial averages at fixed $v$. The Laplace variable $z$ conjugated to $t$ is $z=z^{*} v / \sigma$. In the extreme dilute limit one has $L\left(x, R^{N}\right) \vec{v} \simeq \vec{v}$ and therefore $\hat{\psi}\left(z^{*}\right)$ diverges as $z^{*-1}$. Thus $\hat{\psi}\left(z^{*}\right)$ is written as

$$
\hat{\psi}\left(z^{*}\right)=\left[z^{*}+\gamma\left(z^{*}\right)\right]^{-1},
$$

where $y\left(z^{*}\right)$ incorporates the collision effects. 
This quantity $\gamma\left(z^{*}\right)$ can be expanded in the density,

$$
\gamma\left(z^{*}\right)=\sum_{\ell=1}^{\infty} n^{* \ell} \gamma_{\ell}\left(z^{*}\right)
$$

A complete treatment ${ }^{2.1)}$ gives for the first few $\gamma_{\ell}\left(z^{*}\right)$ the following $r e-$ sults:

$$
\begin{aligned}
& \gamma_{1}\left(z^{*}\right)=-z \beta_{1}\left(z^{*}\right) \\
& \gamma_{2}\left(z^{*}\right)=-z\left[\beta_{2}\left(z^{*}\right)-\beta_{1}^{2}\left(z^{*}\right)\right] \\
& \gamma_{3}\left(z^{*}\right)=-z\left[\beta_{3}\left(z^{*}\right)-2 \beta_{2}\left(z^{*}\right) \beta_{1}\left(z^{*}\right)+\beta_{1}^{3}\left(z^{*}\right)\right] .
\end{aligned}
$$

The function $\beta_{\ell}\left(z^{*}\right)$ is related to the $l$-particle Ursell operator, being equal to the product of the Laplace transform of $\tilde{\beta}_{\ell}\left(t^{*}\right)$ (2.17) and a factor $z-L(x)$, where $L(x)$ is the Liouville operator in the case of free streaming.

The original hope was that the $\gamma_{\ell}\left(z^{*}\right)$ would behave well for $z^{*} \rightarrow 0$ such that eq. (2.22) implies a series expansion for vo/D. The earlier mentioned divergencies $\sim t^{* \ell}$ exhibit themselves as singularities $\sim_{z^{*}}$ in the $\beta_{\ell}\left(z^{*}\right)$. As (2.23) indicates, the relation between $\gamma_{\ell}\left(z^{*}\right)$ and the $\beta_{\ell}\left(z^{*}\right)$ is such that the dominant singularities are subtracted and then $\gamma_{\ell}\left(z^{*}\right)$ possibly stays finite for $z^{*} \rightarrow 0$. Thus one would obtain a power series

$$
v \sigma / D=\sum_{\ell=1}^{\infty} n^{* \ell} \gamma_{\ell}(0)
$$

However, this subtraction mechanism does not work, since also other singularities than the dominant ones occur. As will be shown in the following sections, the density expansion turns out to be in two dimensions

$$
v \sigma / D=c_{1} n^{*}+c_{2}^{\prime} n^{* 2} l n n^{*}+c_{2} n^{* 2}+c_{3}^{\prime \prime} n^{*}\left(l n n^{*}\right)^{2}+\ldots
$$


with

$$
c_{1}=\frac{16}{3}, c_{2}^{\prime}=-\frac{64}{9}, c_{2}=-4.68, c_{3}^{\prime \prime}=24.10,
$$

and in three dimensions

$$
v \sigma / D=b_{1} n^{*}+b_{2} n^{* 2}+b_{3}^{\prime} n^{* 3} \ln n^{*}+\cdots
$$

with

$$
b_{1}=3 \pi, b_{2}=19.05, b_{3}^{\prime}=0.645 .
$$

\subsubsection{First few expansion coefficients}

The calculation of $c_{1}, c_{2}^{\prime}, b_{1}, b_{2}$ and $b_{3}^{\prime}$ will now be outlined, as performed by Van Leeuwen and Weyland $2.1,2.2)$. The function $\beta_{\ell}\left(z^{*}\right)$, introduced in (2.23), is defined in terms of the usual reduced density distribution function $g\left(\vec{q}, R^{l}\right)$ and the collision operator $B_{z}\left(x, R^{l}\right)$ as

$$
\beta_{\ell}\left(z^{*}\right)=\sigma^{-\ell d \frac{\vec{v}}{v^{2}}} \cdot \int \frac{d R^{l}}{l !} g\left(\vec{q}, R^{l}\right) B_{z}\left(x, R^{l}\right) \vec{v},
$$

where $B_{Z}\left(x, R^{l}\right)$ is proportional to the Laplace transform of the Ursell operator of the moving particle and $\ell$ scatterers. To give an example, the so-called binary kernel $B_{z}\left(x, \vec{R}_{i}\right)$, which involves only one scatterer, is defined as

$$
B_{z}\left(x, \vec{R}_{i}\right)=\int_{0}^{\infty} d t e^{-z t}\left[\exp t L\left(x, \vec{R}_{i}\right)-\exp t L(x)\right][z-L(x)]
$$

Two contributions can be recognized in the binary kernel $B_{z}\left(x, \vec{R}_{i}\right)$ : one due to a real collision, $\mathrm{B}_{z}^{\mathrm{r}}\left(\mathrm{x}, \overrightarrow{\mathrm{R}}_{i}\right)$, corresponding to the two-particle operator exp $t L\left(x, \vec{R}_{i}\right)$, and the other due to a virtual collision, $B_{z}^{v}\left(x, R_{i}\right)$, corresponding to the free streaming operator exp $t L(x)$. One 
has obviously

$$
B_{z}\left(x, \vec{R}_{i}\right)=B_{z}^{r}\left(x, \vec{R}_{i}\right)-B_{z}^{V}\left(x, \vec{R}_{i}\right)
$$

To evaluate the coefficients $\gamma_{\ell}\left(z^{*}\right)$ it is necessary, for $l \geqslant 2$, to introduce the binary collision expansion. By this expansion $B_{z}\left(x, R^{l}\right)$ is expressed as an iterated sum over products of the binary kernel, $B_{z}\left(x, \vec{R}_{i}\right)$. This means that the generated collision sequences are characterized by the number of collisions that the moving particle suffers from a certain number of scatterers. One has in particular for $\beta_{2}\left(z^{*}\right)$ :

$$
\beta_{2}\left(z^{*}\right)=\sum_{j=2}^{\infty} \beta_{2, j}\left(z^{*}\right),
$$

where in $\beta_{2, j}\left(z^{*}\right)$ the first subscript denotes the number of scatterers involved and the second one the number of binary kernels, i.e. the nunber of collisions.

Furthermore it is useful to divide the contributions in 'dynamical' and 'statistical' ones:

$$
\beta_{l}\left(z^{*}\right)=\beta_{l}^{d y}\left(z^{*}\right)+\beta_{l}^{s t}\left(z^{*}\right),
$$

with the definitions

$$
\begin{aligned}
& \beta_{\ell}^{d y}\left(z^{*}\right)=\sigma^{-\ell d \frac{\vec{v}}{2}} \cdot \int \frac{d R^{l}}{\ell !} B_{z}\left(x, R^{l}\right) \vec{v} \\
& \beta_{\ell}^{s t}\left(z^{*}\right)=\sigma^{-\ell d \frac{\vec{v}}{2}} \cdot \int \frac{d R^{l}}{\ell !}\left[g\left(\vec{q}, R^{\ell}\right)-1\right] B_{z}\left(x, R^{l}\right) \vec{v} .
\end{aligned}
$$

The statistical (more complicated) part is defined such that it is zero for large distances between the particles. The motivation for this division is that possible divergencies can only originate from contributions at asymptotically large distances and therefore will occur dominantly in the (relatively simple) dynamical part.

For the calculation of $\gamma_{1}\left(z^{*}\right)$, see (2.23), there is no need for the binary collision expansion. In the limit of $z^{*} \rightarrow 0$ there is no contri- 
bution from $\beta_{1}^{\text {st }}\left(z^{*}\right)$, since this quantity remains finite in this limit due to the factor $[g(\vec{q}, \vec{R})-1]$ which is zero for large distances. The dynamical part $\beta_{1}^{d y}\left(z^{*}\right)$ is easily evaluated and substitution in (2.23) leads to

$$
\begin{array}{ll}
\gamma_{1}^{d y}\left(z^{*}\right)= & \text { for } d=2, \\
\pi & \text { for } d=3 .
\end{array}
$$

Inspecting the expression (2.23) for $\gamma_{2}\left(z^{*}\right)$, we observe that contributions arise from both $\beta_{1}\left(z^{*}\right)$ and $\beta_{2}\left(z^{*}\right)$. The first contribution $\beta_{2,2}^{d y}\left(z^{*}\right)$, the dynamical two-scatterers two-collisions contribution, has the property

$$
\beta_{2,2}^{d y}\left(z^{*}\right)=\left[\beta_{1}^{d y}\left(z^{*}\right)\right]^{2},
$$

because the two collisions occur at two independent scatterers (and no statistics is involved). Therefore both the singular terms of (2.36) cancel in (2.23), as was indicated in the last section. The first nonvanishing collision sequence appears through $\beta_{2,3}^{d y}\left(z^{*}\right)$, where the moving particle collides with a first scatterer, then with a second scatterer and again with the first one (see fig. 2.1). This process is divergent in

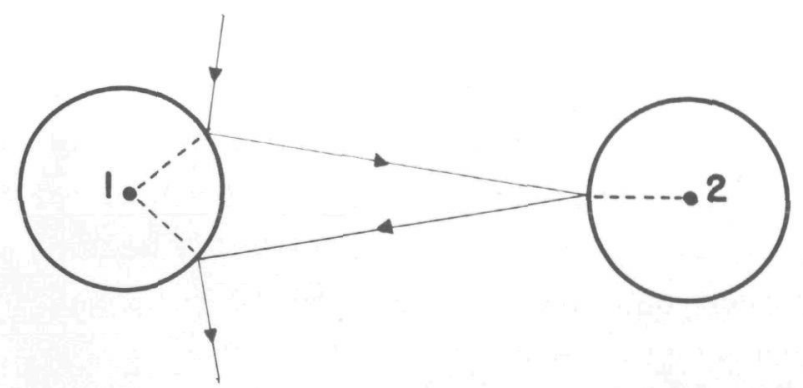

Fig. 2.1. Collision sequence corresponding to $\beta_{2,3}^{d y}\left(z^{*}\right)$, the first and the last collision chosen to be real.

the two-dimensional case for $z^{*} \rightarrow 0$ and leads to 


$$
\gamma_{2,3}^{d y}\left(z^{*}\right)=-\frac{1}{2}\left(\frac{8}{3}\right)^{2} \ln z^{*}+0(1) \text { for } d=2
$$

The second suffix of $\gamma_{\ell, j}\left(z^{*}\right)$ has the same meaning as the one of $\beta_{\ell, j}\left(z^{*}\right)$ (2.32) and counts the number of binary collision operators involved in the diagram.

In order to convert the power series in the density $n^{*}$, which diverges with $z^{*} \rightarrow 0$, into a convergent series, one performs a renormalization summation. In the case mentioned above, $\gamma_{2,3}^{d y}\left(z^{*}\right)$, which is the lowest order ring diagram, one sums over all the higher-order ring diagrams $\gamma_{\ell, \ell+1}\left(z^{*}\right)$ and obtains the so-called ring sum $\gamma_{\Upsilon}\left(z^{*}\right)$ :

$$
\gamma_{r}\left(z^{*}\right)=\sum_{\ell=2}^{\infty} n^{* \ell} \gamma_{\ell, \ell+1}^{d y}\left(z^{*}\right)
$$

This sum is hard to do in general, but fortunately the main interest is in the $n^{*} \rightarrow 0$ behaviour. As a general guide-line for renormalization, which is also of computational value, one can use the following receipt. Take a diagram, e.g. $\gamma_{2,3}^{d y}\left(z^{*}\right)$, where all collisions are real $\left(B_{z}^{r}\left(x, \vec{R}_{i}\right)\right)$, except the first and the last one $\left(B_{z}\left(x, \vec{R}_{i}\right)\right)$, and dress it with all possible virtual collisions $\left(B_{z}^{V}\left(x, \vec{R}_{i}\right)\right)$. As a first approximation the contribution of such a sum is obtained by replacing the parameter $\mathrm{z}^{*}$ by $z^{*}+a_{d} n^{*}$ (see (2.13) for the definition of $a_{d}$ ). Then one may take the limit $z^{*} \rightarrow 0$ and one sees that a $\ln z^{*}$ in the unrenormalized contribution is replaced by a $\ln \mathrm{n}^{*}$ in the renormalized diagram contribution, called $\gamma_{2,3}^{r, d y}\left(z^{*}\right)$. Along these lines the regular contributions can also be obtained, though often with considerable more effort. Weyland and Van Leeuwen ${ }^{2.2)}$ obtained for the ring sum $\gamma_{\Upsilon}\left(z^{*}\right)$ in this way

$$
\gamma_{r}(0)=-\frac{1}{2}\left(\frac{8}{3}\right)^{2} n^{*^{2}} \ln n^{*}+0\left(n^{*}\right) \text { for } d=2 \text {. }
$$

In the three-dimensional case $\gamma_{2}\left(z^{*}\right)$ is finite. Of the dynamical terms only $\gamma_{2,3}^{d y}(0)$ and $\gamma_{2,4}^{d y}(0)$ have been evaluated: 


$$
\begin{aligned}
& \gamma_{2,3}^{d y}(0)=\pi^{2}\left(1 / 3+4 \pi^{2} / 105\right) \\
& \gamma_{2,4}^{d y}(0)=-0.65 \pm 0.01
\end{aligned}
$$$$
\text { for } \mathrm{d}=3 \text {. }
$$

Because of the rapid decrease in the contributions of diagrams with an increasing number of collisions and the same number of scatterers ${ }^{2.2)}$, it was argued by Van Leeuwen and Weyland that the contribution from higher-order terms would only amount to a few percent. The statistical terms are all zero to lowest order in the density for our case of pointscatterers.

The calculation of $\gamma_{3}\left(z^{*}\right)$ does yield a divergent result in three dimensions. The responsible diagrams are $\gamma_{3,4}^{d y}\left(z^{*}\right)$ and $\gamma_{3,5}^{d y}\left(z^{*}\right)$. Their contributions amount to

$$
\begin{aligned}
& \gamma_{3,4}^{d y}\left(z^{*}\right)=\frac{\pi^{3}}{4} \ln z^{*}+0(1) \\
& \gamma_{3,5}^{d y}\left(z^{*}\right)=-\left[\pi^{3} / 4-0.215\right] \text { ln } z^{*}+0(1)
\end{aligned} \text { for } d=3
$$

It is clear from (2.41) that it will not suffice to sum over the ring diagrams $\gamma_{Y}\left(z^{*}\right)$ only to remove these divergencies, but that one also has to sum over a special class of diagrams $\gamma_{S}\left(z^{*}\right)$ corresponding to $\gamma_{3,5}^{d y}\left(z^{*}\right)$. The result of these sums reads

$$
\gamma_{r}(0)+\gamma_{S}(0)=0.215 n^{*^{3}} \ln n^{*}+0\left(n^{*}\right) .
$$

\subsubsection{Higher-order regular contributions in two dimensions}

For the comparison of the computer-experiment results with the diffusion coefficient obtained from the density expansion, which comparison will be performed mainly for the two-dimensional case, it is convenient to know the contributions from terms next to the first singular one. In this section the diagrams of order $n^{*}{ }^{2}$ will be discussed and their contributions will be given. There are four sources 
of regular contributions of order $\mathrm{n}^{2}$ :

$$
\begin{aligned}
& \text { 1) } \lim _{\mathrm{n}^{*} \rightarrow 0} \gamma_{2,3^{r, d y}(0)} \text { regular }=-3.572 \text {, } \\
& \text { 2) } \lim _{\mathrm{n}^{*} \rightarrow 0} l_{l^{=}}^{\infty} \mathrm{n}^{*(\ell-2)} \gamma_{\ell, \ell+1}^{r, d y}(0)=1.020 \text {, } \\
& \text { 3) } \sum_{\ell=4}^{\infty} \gamma_{2, \ell}^{d y}(0)=-1.92 \text {, }
\end{aligned}
$$

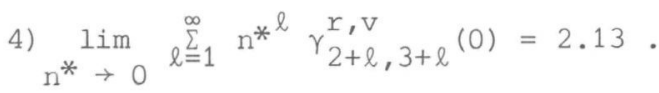

Ad 1) This contribution results from a more complete calculation of a part of the ring sum $\gamma_{Y}(0)$, see (2.38) and (2.39). The logarithmic term in (2.39) is due to the renormalized diagram $\gamma_{2,3}^{r, d y}(0)$. This renormalized diagram also gives a regular contribution of order $\mathrm{n}^{*}{ }^{2}$, in the low-density limit. The calculation is complicated and contains a serie. expansion in terms of Bessel functions. The first term in this expansion can be obtained analytically and yields the logarithmic term and a regular contribution. The other terms in this expansion, all regular, have to be computed numerically. The series converges fast. The first seven terms have been computed to obtain the above mentioned result, the next six terms would contribute by an amount of the order of $10^{-4}$.

Ad 2) The other part of the ring sum contains renormalized ring diagrams of higher order, $\gamma_{\ell, \ell+1}^{r, d y}$ with $\ell \geqslant 3$. At first sight it may be surprising that diagrams with more than two scatterers do contribute to order $n^{* 2}$. Indeed the unrenormalized diagrams $\gamma_{l, l+1}^{d y}$ contribute to order $\mathrm{n}^{*}$, but the renormalization leads to cancellation of $\ell-2$ powers of the density. For the calculation it is convenient to split the sum over the higher-order ring diagrams into two parts. One sum converges slowly, but fortunately can be done analytically, giving a contribution of $64\left(\frac{1}{2}-1 / \pi\right) / 9$. The other sum converges rapidly, successive terms decreasing by about a factor of ten. The result obtained is: $-0.2724 \pm 0.00005$.

Ad 3) This infinite number of diagrams, $\gamma_{2, l}^{d y}(0)$, for $\ell \geqslant 4$, is generated by the binary collision expansion, see (2.32). Together they 
represent all possible collision sequences between the moving particle and two scatterers only, with the total number of collisions $\geqslant 4$. They give a non-zero contribution only when all the collisions are real, except the first and the last collision. No diagram could be reduced further than to a three-dimensional integral. These integrals have been computed numerically. For even $\ell$ the diagrams appear to give negative and for odd \& positive contributions. After 20 terms the absolute value of the terms is reduced to $0.1 \%$ of the first term. The sum of two succeeding terms, of opposite sign, is even smaller. The sum of terms between 20 and 40 indeed appears to be negligible. The succeeding ones will be even more so, especially because the accuracy of the numerical integration of the first terms is not much better than one percent.

Ad 4) This last contribution of order $1^{*^{2}}$ comes from a class of diagrams closely related to the renormalized diagram $\gamma_{2,3}^{r, d y}$. In the renorma-

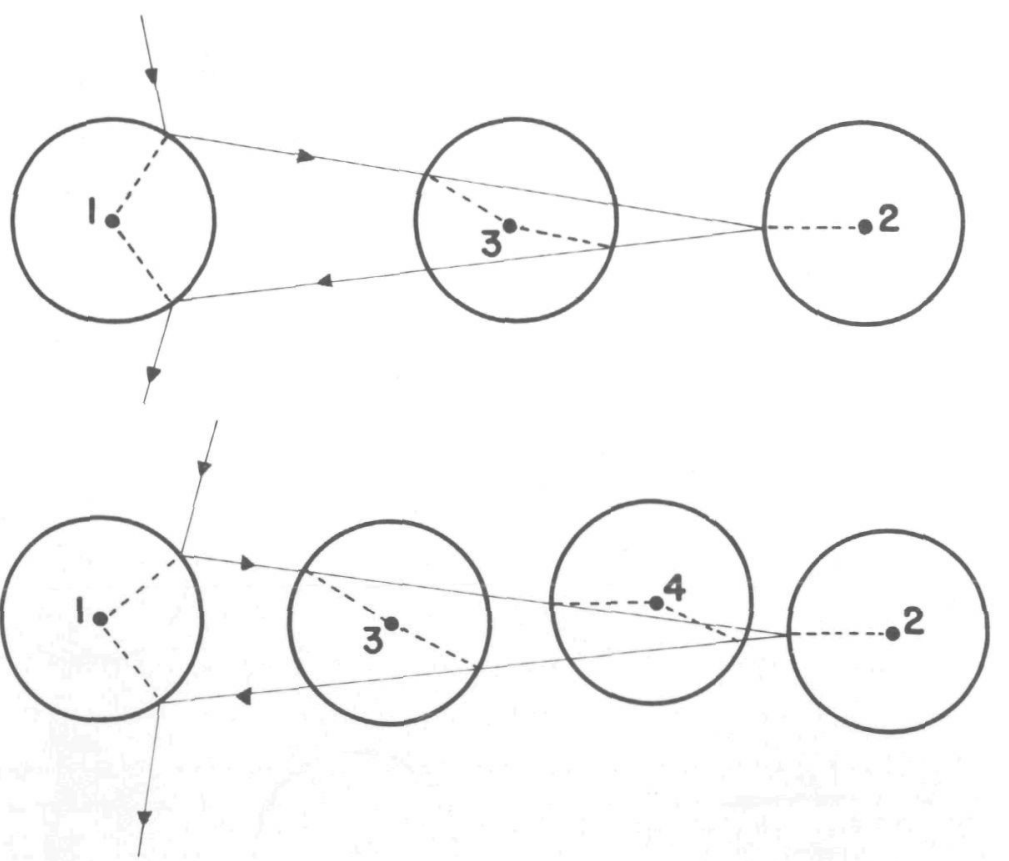

Fig. 2.2. Two diagrams of the special class, the first and the last collisions chosen to be real. 
lization procedure diagrams are 'dressed' with an arbitrary number of intermediate scatterers, undergoing virtual collisions only. It has been argued by Weyland and Van Leeuwen ${ }^{2.2)}$ that the ring diagram $\gamma_{2,3}^{r, d y}$ supplied with intermediate scatterers hit twice virtually gives rise to a special class contributing in qualitatively the same way as $\gamma_{2,3}$, dy itself. The two most simple diagrams out of this special class are shown in fig. 2.2. Taking $\gamma_{2,3}^{r, d y}$ and the special class together one arrives at the same 10garithmic term as given in (2.39). The regular contribution of the special class is easily obtained from the numerical integration of a twodimensional integral.

\subsubsection{Higher-order singular contributions in two dimensions}

The most divergent part of $\gamma_{3}(0)$, which gives rise to $\frac{1}{2} c_{3}^{\prime \prime}\left(\ln n^{*}\right)^{2}$ in the low-density limit after renormalization, consists of quite a number of diagrams. They are listed together with their respective contributions in table 2.1. The first diagram in table 2.1 is shown in fig. 2.3 as an example. Similarly to (2.37) only the 'dynamical' part of the diagrams lead to a density dependence of this kind. It should be noted that $B_{i}$ is a short-hand notation for $B_{z}\left(x, \vec{R}_{i}\right)$. The binary collision operator $B_{z}\left(x, \vec{R}_{i}\right)$ is defined in $(2.30)$ and its real and virtual parts, $B_{z}^{r}\left(x, \vec{R}_{i}\right)$ and $B_{z}^{v}\left(x, \vec{R}_{i}\right)$, in $(2.31)$.

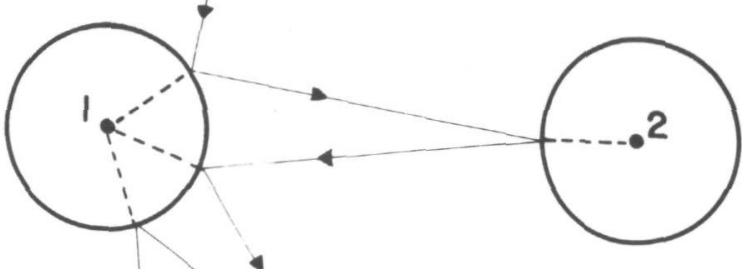

Fig. 2.3. The diagram $B_{1} B_{2}^{r} B_{1}^{r} B_{3}^{r} B_{1}$,

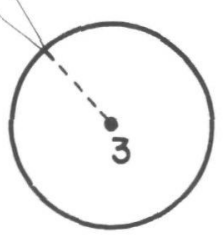
the first and the last collision chosen to be real. 


\begin{tabular}{|c|c|}
\hline Diagram & Contribution \\
\hline $\mathrm{B}_{1} \mathrm{~B}_{2}^{\mathrm{r}} \mathrm{B}_{1}^{\mathrm{r}} \mathrm{B}_{3}^{\mathrm{r}} \mathrm{B}_{1}$ & $(4 / 3)^{2}[(8 / 3)-2]$ \\
\hline $\mathrm{B}_{1} \mathrm{~B}_{2}^{\mathrm{x}} \mathrm{B}_{1}^{\mathrm{V}} \mathrm{B}_{3}^{\mathrm{r}} \mathrm{B}_{1}$ & $(4 / 3)^{2} \quad 2$ \\
\hline$B_{1} B_{2}^{r} B_{3}^{r} B_{1}^{r} B_{3}$ & $(4 / 3)^{2} \quad 1 / 3$ \\
\hline $\mathrm{B}_{1} \mathrm{~B}_{2}^{\mathrm{x}} \mathrm{B}_{1}^{\mathrm{r}} \mathrm{B}_{3}^{\mathrm{r}} \mathrm{B}_{2}$ & $(4 / 3)^{2}$ \\
\hline$B_{1} B_{2}^{x} B_{3}^{x} B_{1}^{x} B_{2}$ & $-(4 / 3)^{2}$ \\
\hline $\mathrm{B}_{1} \mathrm{~B}_{2}^{\mathrm{r}} \mathrm{B}_{3}^{\mathrm{r}} \mathrm{B}_{2}^{\mathrm{r}} \mathrm{B}_{1}$ & $(4 / 3)^{2} \pi / 2$ \\
\hline $\mathrm{B}_{1} \mathrm{~B}_{2}^{\mathrm{V}} \mathrm{B}_{3}^{\mathrm{r}} \mathrm{B}_{2}^{\mathrm{r}} \mathrm{B}_{1}$ & 0 \\
\hline $\mathrm{B}_{1} \mathrm{~B}_{2}^{\mathrm{r}} \mathrm{B}_{3}^{\mathrm{r}} \mathrm{B}_{2}^{\mathrm{V}} \mathrm{B}_{1}$ & 0 \\
\hline $\mathrm{B}_{1} \mathrm{~B}_{2}^{\mathrm{r}} \mathrm{B}_{3}^{\mathrm{V}} \mathrm{B}_{1}^{\mathrm{r}} \mathrm{B}_{3}$ & $(4 / 3)^{2}$ \\
\hline $\mathrm{B}_{1} \mathrm{~B}_{2}^{\mathrm{r}} \mathrm{B}_{1}^{\mathrm{V}} \mathrm{B}_{3}^{\mathrm{r}} \mathrm{B}_{2}$ & $(4 / 3)^{2}$ \\
\hline$B_{1} B_{2}^{r} B_{3}^{r} B_{1}^{V} B_{2}$ & $(4 / 3)^{2}$ \\
\hline $\mathrm{B}_{1} \mathrm{~B}_{2}^{\mathrm{V}} \mathrm{B}_{3}^{\mathrm{r}} \mathrm{B}_{1}^{\mathrm{r}} \mathrm{B}_{2}$ & $(4 / 3)^{2}$ \\
\hline $\mathrm{B}_{1} \mathrm{~B}_{2}^{\mathrm{V}} \mathrm{B}_{3}^{\mathrm{r}} \mathrm{B}_{2}^{\mathrm{V}} \mathrm{B}_{1}^{\mathrm{r}} \mathrm{B}_{2}$ & $(4 / 3)^{2}[3 / 16-1]$ \\
\hline $\mathrm{B}_{1} \mathrm{~B}_{2}^{\mathrm{r}} \mathrm{B}_{1}^{\mathrm{V}} \mathrm{B}_{3}^{\mathrm{r}} \mathrm{B}_{1}^{\mathrm{V}} \mathrm{B}_{2}$ & $(4 / 3)^{2}[3 / 16-1]$ \\
\hline
\end{tabular}

Table 2.1. Diagrams contributing to $\frac{1}{2} c_{3}^{\prime \prime}\left(\operatorname{In} n^{*}\right)^{2}$. 


\subsubsection{Velocity outocorrelation function}

For the analysis of the velocity autocorrelation function it is convenient to have an expression correct to lowest order in the density, the Boltzmann approximation. This can be easily obtained by substituting $(2.35)$ into $(2.21)$, leading to

$$
\psi_{B}\left(t^{*}\right)=\begin{array}{ll}
\exp \left(-8 n^{*} t^{*} / 3\right) & \text { for } a=2 \\
\exp \left(-\pi n^{*} t^{*}\right) & \text { for } d=3 .
\end{array}
$$

Reducing the time $t^{*}$ with respect to the Boltzmann mean free time $\tau^{*}$, this gives

$$
\phi_{B}(s)=\begin{array}{ll}
\exp -4 s / 3 & \text { for } d=2 \\
\exp -s & \text { for } d=3
\end{array} .
$$

The main interest in the velocity autocorrelation function itself lies in its behaviour for long times. On the basis of kinetic theory Ernst and Weyland ${ }^{2.3)}$ derived the asymptotic behaviour of the velocity autocorrelation function for long times:

$$
\phi_{A}(s)=\begin{array}{ll}
-n^{*} / \pi s^{2} & \text { for } d=2 \\
-(3 \pi)^{\frac{3}{2}} n^{* 2} / 16 s^{\frac{5}{2}} & \text { for } d=3 .
\end{array}
$$

The results hold to lowest order in the density and originate from the ring diagrams (which go beyond the Boltzmann approximation). They differ from the results for fluids with moving particles only $\left(\phi_{A}(s) \sim s^{-d / 2}\right.$ ) by the fact that the long-time tail has a negative sign and contains an additional factor $\mathrm{s}^{-1}$. This also means that it will be possible to inte- 
grate the velocity autocorrelation function up to the infinitely long times, in order to obtain the diffusion coefficient.

\subsection{Free-time distribution}

The subject of this section is not essential for the main objective of this thesis: the density dependence of diffusion. Apart from the fact that the Boltzmann mean free time is a useful scaling quantity, the calculation of the distribution of free times and the mean free time has been undertaken to provide a check on the machine calculations. Initially it was thought that the free-time distribution could be calculated exact$\left.1 y^{2} \cdot 4\right)$. However, the experimental result turns out to differ from the theoretical result. As will be shown in this section, this can be attributed to complications in the theoretical concepts involved.

For the first free time, the distribution and the mean can be easily derived. The first free time $t_{1}\left(x, R^{N}\right)$ is defined as the time until the first collision takes place in a configuration of $\mathrm{N}$ scatterers $\mathrm{R}^{\mathrm{N}}$, the initial position and momentum of the moving particle being specified by $x=(\vec{p}, \vec{q})$. The probability density $P_{N}(t)$ for finding a $t_{1}=t$ is easily expressed in terms of the Dirac $\delta$-function:

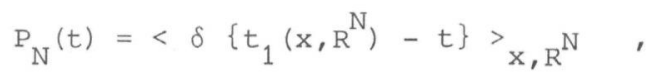

where the average has to be taken over all allowed points in phase space on the energy surface corresponding to velocity $v$. It is convenient for the derivation to introduce the quantity

$$
A_{N}(t)=\int_{t}^{\infty} P_{N}\left(t^{\prime}\right) d t^{\prime},
$$

which is a measure for finding a $t_{1}>t$. Performing the time integral in (2.48) after substitution of $(2.47)$ and writing the ensemble average explicitly one has 


$$
A_{N}(t)=\int \ldots \int_{V} d x d R^{N} H\left\{t,\left(x, R^{N}\right)-t\right\} / \int \ldots \int_{V} d x d R^{N},
$$

where $H$ is the Heaviside function $(H(t)=0$ for $t<0, H(t)=1$ for $t \geqslant 0$ ) and the subscript $\mathrm{v}$ denotes that the integration over velocity space has to be performed at constant $\mathrm{v}$. The integrand in the numerator is only different from zero when none of the scatterers is in a volume of base $a_{d} \sigma^{d-1}$ and height vt attached to the moving particle (again: $a_{1}=1$, $\mathrm{a}_{2}=2$ and $\left.\mathrm{a}_{3}=\pi\right)$. If the volume of the moving particle $\Omega_{\mathrm{m}}\left(\Omega_{\mathrm{m}}=2 \sigma\right.$ for $\mathrm{d}=1, \Omega_{\mathrm{m}}=\pi \sigma^{2}$ for $\mathrm{d}=2$ and $\Omega_{\mathrm{m}}=4 \pi \sigma^{3} / 3$ for $\mathrm{d}=3$ ) is taken into account by introducing a corrected volume $\Omega^{\prime}=\Omega-\Omega_{\mathrm{m}^{\prime}}$ one obtains by performing the integrations

$$
A_{N}(t)=\left(\Omega^{\prime}-\operatorname{vta}_{d} \sigma^{d-1}\right)^{N} / \Omega^{\prime}{ }^{N} .
$$

Using the Boltzmann mean free time $\tau=\left(\text { nva }{ }_{d} \sigma^{d-1}\right)^{-1}$ or rather the Boltzmann mean free time for a system of $\mathrm{N}$ scatterers

$$
\tau_{\mathrm{N}}=\tau\left(1-\mathrm{n} \Omega_{\mathrm{m}} / \mathrm{N}\right),
$$

eq. (2.50) can be rewritten as

$$
A_{N}(t)=\left(1-t / \tau_{N}\right)^{N}
$$

By differentiation $P_{N}(t)$ is obtained

$$
P_{N}(t)=\tau_{N}^{-1}\left(1-t / \tau_{N}^{N}\right)^{N-1}
$$

and in the limit of an infinite system one has

$$
P(t) \equiv \lim _{N \rightarrow \infty} P_{N}(t)=\tau^{-1} \exp (-t / \tau)
$$


By taking the first moment of this distribution function one finds that the average of the first free time is equal to the Boltzmann mean free time:

$$
\lim _{N \rightarrow \infty}\left\langle t_{1}\right\rangle x, R^{N}=\tau
$$

For the distribution of free times, considering now the free times after the first one as well, the situation is more complicated. For the onedimensional case wood ${ }^{2.5}$ derived an explicit expression for the distribution of free times. This is not a pure exponential and the mean free time deviates from the Boltzmann mean free time by a factor two. In appendix $A$ the joint distribution of the first and the second free time is calculated for the two-dimensional case. Here too, the distribution of the second free time is not exponential and the average second free time deviates from the Boltzmann value.

In a previous paper ${ }^{2.4)}$ it has been derived that there is a simple connection between the distribution of the first free time and that of the free times in general. However, this requires a special way of taking averages over different configurations. It is unfeasable to determine the free-time distribution by calculating the third-, fourth-, etc. free-time distribution and taking the average, in view of the complexity of the second-free-time distribution. Below a weighted distribution function of free times will be derived having exact correspondence with the (exponential) distribution of first free times.

Let $M_{T}\left(t ; x, R^{N}\right)$ dt be defined as the number of free times between $t$ and $t+d t$ when the moving particle is followed for a time $T$, starting at $x$ in a configuration of scatterers $R^{N}$. Since each of the free times larger than $t$ on the trajectory contains one first free time of value $t$, one has the equality

$$
\int_{t}^{\infty} M_{T}\left(t " ; x, R^{N}\right) d t^{\prime \prime}=\int_{0}^{T} \delta\left(t_{1}\left\{x\left(t^{\prime}\right), R^{N}\right\}-t\right) d t^{\prime}
$$




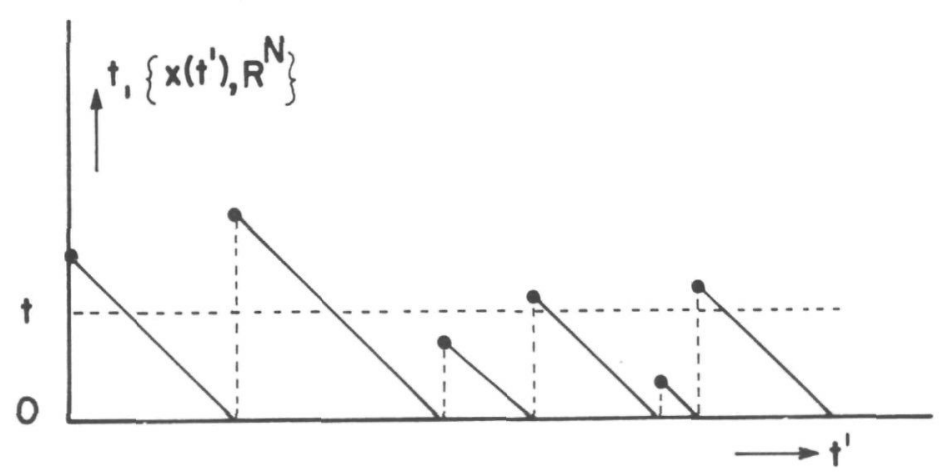

Fig. 2.4. The first free time $t_{1}$ as a function of the trajectory time $t^{\prime}$. The discontinuities are caused by collisions. Each dot represents a free time. Each free time larger than $t$ contains one first free time equal to $t$.

This is illustrated in fig. 2.4, where it should be noted that each value $x\left(t^{\prime}\right)$, on the trajectory, is a valid starting point for a first free time. The integration from 0 to $T$ on the right-hand side is enclosed in $M_{T}\left(t " ; x, R^{N}\right)$ by definition. Furthermore $x\left(t^{\prime}\right)$ is a dynamical variable, $x=(\vec{p}, \vec{q})$, with the momentum $\vec{p}$ changing discontinuously due to collisions and $\vec{q}$ changing continuously with time because of free streaming.

We want to make use of ergodicity but care must be taken because of the occurrence of pockets. Let a pocket in a configuration $R^{N}$ be defined as a region which is enclosed on all sides by scatterers (in the picture that the scatterers are overlapping particles of radius $\sigma$ and the moving particle is a point-particle). In a configuration $\mathrm{R}^{\mathrm{N}}$ without pockets, ensemble and time averages are equal:

$$
\left\langle\delta\left(t_{1}\left\{x, R^{N^{\prime}}\right\}-t\right)\right\rangle_{x}=\lim _{T \rightarrow \infty} \frac{1}{T} \int_{0}^{T} \delta\left(t_{1}\left\{x\left(t^{\prime}\right), R^{N}\right\}-t\right) d t^{\prime},
$$

where $\langle\ldots\rangle_{x}$ denotes the average over all possible $x$, keeping $|\vec{p}|$ fixed. In a configuration with pockets this equality holds for each pocket separately. The average over all configurations $\mathrm{R}^{\mathrm{N}}$, to be taken below, has to be extended by an average over all pockets in that case. Since 
this aspect merely complicates the notation, it will not be taken into account in the following.

Corresponding to $\mathrm{P}_{N}(t)$ a distribution function of first free times for one configuration can be defined: $P\left(t ; R^{N}\right)$. From an equation similar to $(2.47)$,

$$
P\left(t ; R^{N}\right)=\left\langle\delta\left(t_{1}\left\{x, R^{N}\right\}-t\right)\right\rangle_{x},
$$

one finds with $(2.56)$ and $(2.57)$

$$
P\left(t ; R^{N}\right)=\lim _{T \rightarrow \infty} \frac{1}{T} \int_{t}^{\infty} M_{T}\left(t " ; x, R^{N}\right) d t "
$$

In the computer experiment a histogram is obtained which gives the relative frequency $\mathrm{m}_{\mathrm{r}}\left(t_{\mathrm{k}} ; \mathrm{x}, \mathrm{R}^{\mathrm{N}}\right)$ in interval $\mathrm{k}$ :

$$
m_{T}\left(t_{k} ; x, R^{N}\right)=\left[N_{T}\left(x, R^{N}\right)\left(t_{k}-t_{k-1}\right)\right]_{t_{k-1}}^{-1} t_{k} M_{T}\left(t " ; x, R^{N}\right) d t^{\prime \prime} .
$$

Here $\mathrm{N}_{\mathrm{T}}\left(\mathrm{x}, \mathrm{R}^{\mathrm{N}}\right)$ is the number of collisions occurring on the trajectory and the result is made independent of the width of the interval, $\left(t_{k}-t_{k-1}\right)$, by dividing by the width. The mean free time $\tau_{T}\left(x, R^{N}\right)$ of a trajectory in a configuration $R^{N}$, the moving particle being followed for a time $T$ starting at $x$, is defined by the relation

$$
\tau_{T}\left(x, R^{N}\right)=T / N_{T}\left(x, R^{N}\right)
$$

Elimination of $\mathrm{M}_{\mathrm{T}}\left(t^{\prime \prime} ; \mathrm{x}, \mathrm{R}^{\mathrm{N}}\right)$ from (2.59) and (2.60) gives

$$
\lim _{T \rightarrow \infty} m_{T}\left(t_{k} ; x, R^{N}\right) / \tau_{T}\left(x, R^{N}\right)=\left[P\left(t_{k-1} ; R^{N}\right)-P_{t}\left(t_{k} ; R^{N}\right)\right] /\left(t_{k}-t_{k-1}\right) .
$$

The average over all possible configurations is

$$
\lim _{T \rightarrow \infty} m_{T}\left(t_{k} ; x, R^{N}\right) / \tau_{T}\left(x, R^{N}\right)>{ }_{R}^{N}=\left[P_{N}\left(t_{k-1}\right)-P_{N}\left(t_{k}\right)\right] /\left(t_{k}-t_{k-1}\right) .
$$

It should be noted that pockets can be taken into account by including 
an average over $\mathrm{x}$ on the left-hand side. An explicit expression for the right-hand side follows from substitution of (2.53) or, in the limit $\mathrm{N} \rightarrow \infty$, of (2.54). Thus the experimental (measurable) left-hand side of eq. (2.63) should be equal to the theoretical right-hand side (2.63).

Some additional remarks should be made regarding the mean free time. For the derivation of (2.13) the expression for the collision rate $\Gamma$ was inverted to give the Boltzmann mean free time. Because the scatterers are point-particles, this was originally believed to be the correct mean free time. As will be shown in chapter 4 the experimental mean free time shows significant deviations from this expression at high densities. It was pointed out by Wood ${ }^{2.5)}$, that the problem arises because averaging and inversion are non-commuting operations and for higher densities this apparently demonstrates itself. So one cannot expect in general that the average, over different configurations, of the mean free time per configuration (inverse collision rate) is equal to the mean free time calculated by inverting the average collision rate. More explicitly, one can write for the first average $\tau_{e}$ :

$$
\tau_{e}=N_{C}^{-1} \quad \sum_{=1}^{N} T / N_{T}(\alpha)=T N_{C}^{-1} \underset{\alpha=1}{\sum_{C}^{C}} N_{T}^{-1}(\alpha),
$$

denoting different configurations $\left(x, R^{N}\right)$ by $\alpha$ and averaging over $N_{C}$ configurations. The correct average ' ${ }_{e}^{\prime}$ is given by

$$
\tau_{e}^{\prime}=N_{C}^{-1} \sum_{\alpha=1}^{N} T / N_{C}^{-1} \sum_{\alpha=1}^{N} N_{T}^{C}(\alpha)=T / N_{C}^{-1} \sum_{=1}^{N} N_{T}^{C}(\alpha) .
$$

The same problem is met in averaging the distribution of free times. Analogous to $(2.64)$ a straightforward average, $q_{e}\left(s_{k}\right)$, over configurations can be defined in terms of the normalized distribution per configuration $\mathrm{m}_{\mathrm{T}}\left(\mathrm{s}_{\mathrm{k}} ; \alpha\right)$ :

$$
\mathrm{q}_{\mathrm{e}}\left(\mathrm{s}_{\mathrm{k}}\right) \equiv \mathrm{N}_{\mathrm{C}}^{-1} \stackrel{\sum_{=1}^{\mathrm{N}}}{\mathrm{N}^{\mathrm{C}}} \mathrm{m}_{\mathrm{T}}\left(\mathrm{s}_{\mathrm{k}} ; \alpha\right)
$$

Analogous to (2.65) an alternative average $\mathrm{q}_{\mathrm{e}}^{\prime}\left(\mathrm{s}_{\mathrm{k}}\right)$ can be defined 


$$
\begin{aligned}
q_{e}^{\prime}\left(s_{k}\right) & \equiv{ }_{\alpha=1}^{\sum^{C}} m_{T}\left(s_{k} ; \alpha\right) N_{T}(\alpha) / \sum_{\alpha=1}^{N} N_{T}(\alpha)= \\
& =\left(\tau_{e}^{\prime} / T\right) N_{C}^{-1} \sum_{=1}^{N} m_{T}\left(s_{k} ; \alpha\right) N_{T}(\alpha)
\end{aligned}
$$

It is easily verified that the alternative average over the distributions is in correspondence with the left-hand side of (2.63) and only differs from it by a factor '

It is worth mentioning here how the alternative average affects the results for the one-dimensional system. In the first place it yields the correct Boltzmann value for the mean free time. Secondly it gives a pure exponential behaviour for the free-time distribution.

\subsection{Finite-size effects}

One of the main concerns in computer experiments simulating infinite systems is always the size of the system, which is here determined by the number of scatterers $\mathrm{N}$. The $\mathrm{N}$-dependence can rarely be analysed rigorously and practically always it has to be done experimentally. The results of such an analysis will be discussed in section 4.3 . Yet, it would be very convenient to have beforehand an estimate of the $\mathrm{N}$-dependence as a function of the density.

The clearest defect a small system can show is that the moving-particle passes through the system without undergoing collisions. By the application of periodic boundary conditions this will partially be remedied, but not completely because in some cases the moving particle may continue to walk through the system without collisions (channelling). So the number of free times larger than the time $t_{L}^{*}$, needed by the moving particle to travel across the system, should be kept small.

Let us assume that the free-time distribution is identical to the distribution of first free times, eq. (2.54), which is a reasonable approximation for long times as follows from the appendix and the experimental results. Then, the fraction $f$ of the free times larger than 


\section{$1 / \mathrm{d}$}

$\mathrm{t}_{\mathrm{L}}^{*}\left(=\mathrm{L}^{*}=\Omega / \sigma\right)$ can be computed. This gives for two and three dimensions respectively:

$$
f=\exp -t_{L}^{*} / \tau^{*}=\quad \begin{array}{ll}
\exp -2\left(\mathrm{NI}^{*}\right)^{\frac{1}{2}} & \text { for } \mathrm{d}=2 \\
\exp -\pi \mathrm{N}^{\frac{1}{3}} \mathrm{n}^{*} & \text { for } \mathrm{d}=3 .(2.68)
\end{array}
$$

So one needs larger $\mathrm{N}$ for smaller densities to keep $f$ below a certain value. Some of the highest values for $f$ are for the two-dimensional systems studied: $f=1.8 \times 10^{-3}$ for $N=2000$ and $n^{*}=0.005, f=7.2 \times 10^{-7}$ for $N=500$ and $n^{*}=0.1$. For the three-dimensional case one has: $f=0.14$ for $N=100$ and $n^{*}=0.05, f=0.16$ for $N=2000$ and $n^{*}=0.01$.

In the three-dimensional case the given values of $f$ are rather high, but not all the free times contained in $f$ lead to erroneous contributions. One special event that does give errors consists of successive collisions of the moving particle with a scatterer and one of its nearest images across the periodic boundary. For this situation a geometrical factor can be calculated, determined by the angles under which a scatterer sees its periodic images. Multiplication of $\mathrm{f}$ with this geometrical factor gives the probability that this special event takes place. Assuming a random orientation of the velocity aftex the first collision, one finds for the geometrical factor $\mathrm{f}_{\mathrm{g}}$ in the two-dimensional case: $\mathrm{f}_{\mathrm{g}}=2 \times 10^{-3}$ for $\mathrm{N}=2000, \mathrm{n}^{*}=0.005$ and $\mathrm{f}_{\mathrm{g}}=2 \times 10^{-2}$ for $\mathrm{N}=500, \mathrm{n}^{*}=0.1$. The product of $f$ and $f_{g}$ gives an extremely small probability for this special event in both situations. In three dimensions one finds for the geometrical factor: $\mathrm{f}_{\mathrm{g}}=1 \times 10^{-2}$ for $\mathrm{N}=100, \mathrm{n}^{*}=0.05$ and $\mathrm{f}_{\mathrm{g}}=4 \times 10^{-4}$ for $\mathrm{N}=2000$, $\mathrm{n}^{*}=0.01$.

Other events like travelling repeatedly across the system without collisions are not so easily handled. The results in section 4.3 indicate that their influence is dominant. However, the most important result of this section is that once it has been established that within a given accuracy a system is large enough for a certain density, it will be the more so for higher densities. 
References

2.1) Van Leeuwen, J.M.J. and Weyland, A., Physica 36 (1967) 457

2.2) Weyland, A. and van Leeuwen, J.M.J., Physics 38 (1968) 35

2.3) Ernst, M.H. and Weyland, A., Phys. Letters 34A (1971) 39

2.4) Bruin, C., Physica 72 (1974) 261

2.5) Wood, W.W., private communication 


\section{METHOD OF COMPUTATION}

\subsection{Monte Carlo method}

In the computer experiment one has to simulate for a given density $\mathrm{n}^{*}$ an infinite system of point-scatterers with one moving particle of radius $\sigma$. The trajectory of the moving particle has to be computed, starting from a given position with given velocity, and the average over all possible initial conditions of the moving particle has to be performed. This has been approximated by generating many different configurations of a finite number of scatterers $N$ and by computing the trajectory of the moving particle in each configuration for one initial condition. The number $\mathrm{N}$ should be large enough to make finite-size effects sufficiently small.

Since the scatterers are point-particles, the configurations can be obtained from a crude Monte carlo method: the coordinates should be chosen from random numbers uniformly distributed on the interval (0, $\left.L^{*}\right)$. This holds only partly for the coordinates of the moving particle since they should not give overlap with one of the scatterers.

The (pseudo-) random number generator used is RANDU from IBM ${ }^{3 \cdot 1}$ ). This is a multiplicative congruential generator, so it generates a random integer $U_{i+1}$ from $U_{i}$ according to

$$
\mathrm{U}_{\mathrm{i}+1}=\mathrm{a} \cdot \mathrm{U}_{\mathrm{i}} \text { modulo } \mathrm{M}
$$

where one has for the multiplier $a=65539=2^{16}+3$ and for the modulus $M=2^{31}$, the exponent being equal to the word size of the machine minus one sign-bit.

Many tests on the randomness of numbers produced by random generators have been developed, see e.g. Knuth $^{3 \cdot 2)}$. Multiplicative congruential 
generators for which a is of the order of $\sqrt{ } \mathrm{M}$ pass quite a number of tests ${ }^{3.3)}$. The tests on uniformity and sexial correlation have been performed by the authox on the generator used here (3.1), showing random behaviour with high accuracy. However, congruential generators do show serious deviations from randomness with respect to d-tuples, being important for the case that consecutive (pseudo-) random numbers are used as coordinates in d-dimensional space. This has been found by Macharen and Marsaglia ${ }^{3.3)}$ for triples $(d=3)$ and this feature has been explained by Marsaglia ${ }^{3.4)}$ by showing that 'random numbers fall mainly in plains'. The upper bound for the number of plains containing all d-tuples appears to decrease rapidly with $d$. Some tests sensitive in this respect are the $r^{2}$ test ${ }^{3.5}$ ) and the test on d-dimensional uniformity. In the $r^{2}$ test one computes the distribution of distances $r$ occurring in a d-dimensional cube between points having (pseudo-) random numbers as coordinates. In a test on d-dimensional uniformity the ddimensional cube is divided in smaller cubes (cells) and the occupation numbers of the cells are computed.

The distribution of free times and the mean free time, obtained from the trajectory of a moving particle in a configuration of scatterers can also provide a check on the randomness of a configuration. Though there are some problems in the theoretical expressions for these quantities (section 2.3), they turned out to be useful.

For the 2-dimensional configurations the coordinates of the scatterers $\left(x_{i}, y_{i}\right)$ have been obtained from the generator (3.1) consecutively:

$$
x_{i}=U_{2 i-1} L^{*} / M, \quad y_{i}=U_{2 i} L^{*} / M .
$$

Although this way of generation suffers from the above-mentioned defect, the number of planes containing all the positions appears to be sufficiently large to let the generator pass all three tests, even if the width of the interval in the $r^{2}$ test or the cell-size in the 2-dimensional uniformity test is made small (of the order of $L^{*} / 100$ to $L^{*} / 1000$ ).

For the 3-dimensional configurations the strategy of eq (3.2) 


$$
x_{i}=U_{3 i-2} L^{*} / M, \quad y_{i}=U_{3 i-1} L^{*} / M, \quad z_{i}=U_{3 i} L^{*} / M,
$$

leads to configurations failing all three tests, thus being significantly non-random. Another way of obtaining the coordinates from the same generator,

$$
x_{i}=U_{i} L^{*} / M, \quad y_{i}=U_{N+i} L^{*} / M, \quad z_{i}=U_{2 N+i} L^{*} / M,
$$

turned out to give satisfactory results for all three tests. Therefore eq. (3.4) has been used for the generation of 3-dimensional configurations, see also R. Amadori ${ }^{3 \cdot 6)}$.

\subsection{Molecular-dynamics method.}

The subject of this section is the solution of the equation of motion of the moving particle in a given configuration of scatterers. In addition to the generation of positions (section 3.1) the initial velocity of the moving particle and the boundary conditions have to be specified. As usual in this type of computations periodic boundary conditions are imposed on the system. In this case they result in a model of one particle moving in an infinite lattice with the chosen configuration of scatterers as unit cell. The magnitude of the velocity is a constant of motion and is used as a scaling quantity. The initial direction is chosen at random.

It follows from the hard-sphere interaction, eq. (2.2), that the moving particle undergoes instantaneous collisions, changing its velocity in direction, and that it moves freely between the collisions. The algorithm for this type of motion is a simplification of the one already described by Alder and wainwright ${ }^{3.7)}$. The relation between the velocities before and after a collision, $\overrightarrow{\mathrm{v}}$ and $\overrightarrow{\mathrm{v}}$ ' respectively, reads

$$
\vec{v}^{\prime}=\vec{v}-2\left(\vec{v} \cdot \vec{\sigma}_{i}\right) \vec{\sigma}_{i} / \sigma^{2},
$$

where one has $\vec{\sigma}_{i}=\vec{q}-\vec{R}_{i}$, $\vec{q}$ denoting the position of the moving particle 
at the moment it collides with scatterer $i$ at position $\vec{R}_{i}$. For the determination of the moment $t_{i}$ of the collision between the moving particle starting from $\vec{q}(0)$ and scatterer $i$ at $\vec{R}_{i}$, assuming that the other scatterers do not interfere, one uses the equation

$$
\vec{q}(0)+\vec{v}_{i}-\vec{R}_{i}=\vec{\sigma}_{i}
$$

The solution for the time $t_{i}$ from this equation, which can be obtained after taking the square, reads

$$
t_{i}=-\vec{v} \cdot\left(\vec{q}(0)-\vec{R}_{i}\right) / v^{2} \pm\left[\left\{\vec{v} \cdot\left(\vec{q}(0)-\vec{R}_{i}\right) / v^{2}\right\}^{2}-\left\{\left(\vec{q}(0)-\vec{R}_{i}\right)^{2}-\sigma^{2}\right\} / v^{2}\right\}^{\frac{1}{2}} .
$$

This may result in (see fig. 3.1):

1) no real roots (no collision),

2) negative roots (collision in the past),

3) one or two positive roots (the smallest root is the physical one).

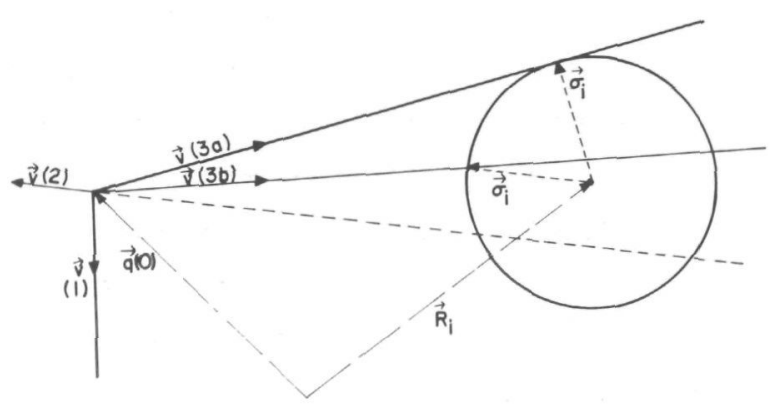

Fig. 3.1. Solutions of eq. (3.6). The indices correspond to the cases mentioned below eq. (3.7).

Starting from an initial state $(\vec{q}(0), \vec{v})$ the smallest positive $t_{i}$ $(i=1,2, \ldots, N)$ determines the next collision. The particle freely moves for a time $t_{i}$, the change in direction of the velocity due to the collision is computed and the procedure is repeated. The computation of $t_{i}$ for all scatterers before everycollision demands a lot of machine-time. 
However, the computing time can be reduced by considering only the scatterers in the neighbourhood of the moving particle. For this purpose a grid is imposed on the system, with a cell size that is considerably smaller than the volume of the system. This of course raises the problem that the moving particle could miss a collision with a scatterer lying in an adjacent cell.

One way to overcome this problem is to enlarge the cells by a region of width $\sigma$ in all the coordinate-directions, thus giving the cells an overlapping region of width $2 \sigma$. These enlarged cells can now be treated separately. A dummy free time is introduced which represents the time it takes for the moving particle to reach the boundary of its cell. If the moving particle does not collide with one of the scatterers of its cell before it crosses the boundary at some position, that position is taken as a new starting point and the procedure is repeated in the adjacent cell. In enlarging the cells the periodic boundary conditions have to be taken into account. During the motion these conditions can easily be handled by computing the number of the adjacent cell properly. This method has been used for the computations in two dimensions.

For the 3-dimensional case this method was too extensive to program and therefore another method was chosen which is equally efficient. In that method there is no overlap between the cells. The problem of missing a collision is solved by considering all surrounding cells (there are 26 of them, in three dimensions) together with the cell in which the moving particle is. In this cube of 27 cells a cube is defined smaller by an amount of $\sigma$ in all coordinate-directions. The smallest free time found within this smaller cube determines the next collision. After performing the collision the whole procedure is repeated, if nccessary in an adjacent cell. If the computed next collision would have to take place outside the smaller cube, there is no guarantee that it is indeed the next one. The particle is moved to the boundary of the adjacent cell and the whole procedure is repeated. If no collision is found with any of the scatterers within the 27 cells, the particle is moved to the boundary of its cell and only a limited number of cells (9) have to be considered in the following step. To facilitate the handling of the periodic boundary conditions in 
this case, the system is enlarged with a layer of a thickness of one cell in all coordinate-directions.

In both methods the optimal number of cells depends upon the number of scatterers and also the density. In the first method the lower bound on the number of cells is trivial, being 1 , and the minimum length of a cell is $2 \sigma$. In the second method the lower bound on the number of cells is 27 and the minimum length of a cell is $\sigma$.

\subsection{Correlation functions}

The numerical solution of the equation of motion results in the velocity $\vec{v}(s)$ and the position $\vec{q}(s)$ of the moving particle as a function of the reduced time $s=t / \tau$, measured with respect to the initial state of the moving particle. The correlation functions at a certain density are obtained as an average over $\mathrm{N}_{f}$ correlation sequences for one configuration of scatterers and an average over the results of $\mathrm{N}_{\mathrm{C}}$ configurations. The normalized velocity autocorrelation function of the moving particle is

$$
\phi\left(s_{\ell}\right)=\frac{1}{N_{C} N_{f}} \quad j=1 \sum_{i=1}^{N_{C}} \quad \vec{v}\left(s_{i}\right) \cdot \vec{v}\left(s_{i}+s_{\ell}\right) / v^{2},
$$

where the dependence of $\vec{v}$ on configuration $j$ is not indicated explicitly. Similarly the mean square displacement is obtained as

$$
\Delta\left(s_{\ell}\right)=\frac{1}{N_{C} N_{f}} \quad \sum_{j=1}^{N_{C}} \sum_{i=1}^{N_{f}}\left(\vec{q}\left(s_{i}+s_{\ell}\right)-\vec{q}\left(s_{i}\right)\right)^{2} / \sigma^{2} .
$$

The integer $l$ runs from zero to a maximum value $l_{\max }$ and one has $s_{\ell}=h l, h$ being the time increment with which the correlation function is sampled. The starting time of correlation sequence $i$ is $s_{i}$ on the absolute time scale.

It saves computing time for the solution of the equation of motion if the delay $s_{i}-s_{i}-1$ between two succeeding correlation seguences, 
$i-1$ and $i$, is taken much smaller than the maximum time $h l_{\max }$ for which the correlation sequence is sampled. On the other hand if one would take the delay as small as the time increment $h$, the information is still so much correlated that succeeding correlation sequences scarcely improve the statistical accuracy. The time-celay $s_{i}{ }^{-s_{i-1}}$ is thus to be chosen as a compromise between minimizing the total time the moving particle is followed in a configuration and minimizing the number of correlation sequences needed to obtain a certain statistical accuracy. The value $s_{i}{ }^{-s_{-1}}=\frac{1}{2}$, measured in units of $\tau$, has been adopted in many cases; other values being $\frac{3}{4}$ and 1 .

In the machine program, the values of a number of correlation sequences at their respective $s_{\ell}$ are computed at a certain time $s$, where $l$ follows from $l=s_{\ell} / h=\left(s-s_{i}\right) / h$ for each sequence $i$. One is only interested in values of $l$ which satisfy the condition $0<\ell<l_{\max }$. This condition determines the correlation sequences which are to be considered at time $\mathbf{s}$. For each sequence the starting conditions $\vec{q}\left(s_{i}\right)$ and $\vec{v}\left(s_{i}\right)$ are still kept in memory at $\mathbf{s}$. In this way of programming it saves time to have the time-delay $s_{i}-s_{i-1}$ as a multiple of $h$.

Another way of computing correlation functions can be found from the observation that the average over $i$ in eq. (3.8) is a discrete convolution, as was pointed out by Futrelle and McGinty ${ }^{3.8)}$. By taking discrete fourier transforms the number of operations can be reduced considerably, especially when the fast-fourier-transform (FFT) technique is used. However, for the low-density calculations that are our main objective, this method turned out to take more machine-time on comparison. The reason for this can easily be understood from the observation that the time-delay between two succeeding functions in the FFT method is inherently equal to the time increment $h$. So this method samples less efficiently than the method described before. Moreover there is the disadvantage that the statistical accuracy of the correlation function decreases for longer times. The situation is even worse in the case of high densities where the velocity autocorrelation function has a long negative tail. In the method used here it is possible to work with different time increments at the same time, a small one for the region where the function decays fast and a large one to sample the tail which varies slowly. An efficient choice for the long-time increment is the delay time, $s_{i} s_{i-1}$. 


\subsection{Diffusion coefficients}

The numerical integration of the velocity autocorrelation function (eq. (2.14)) to obtain the 'experimental' diffusion coefficient is restricted in its accuracy because of the time increment $h$ adopted and because of the finite time hl ${ }_{\text {max }}$ for which the correlation function is considered. The first point could be settled satisfactorily by taking $h=0.1$ or $h=0.15$. Then the error due to the size of $h$ is smaller than the statistical error. In many cases, when the statistical error was not extremely small, $h=0.2$ could be taken as well. The error due to the size of $h$ could be estimated by using different integration rules, or alternatively by applying one integration rule for a number of values for $\mathrm{h}$ and extrapolating these results to $\mathrm{h}=0$. All results to be mentioned later have been obtained with Simpson's integration rule. The second point cannot be settled so easily, because the required value of $l_{\max }$ depends upon the density and hence cannot be given in general. The numerical differentiation of the mean square displacement has been replaced by a least-squares fit of a straight line, the slope of which is proportional to the diffusion coefficient (eq. (2.15)). The time increment for the mean square displacement should not be chosen too small since succeeding points would then be highly correlated. Values that have been used are $h=0.5$ and $h=1$. Another important value is ' $\ell_{\text {ax' }}$ the choice of which is closely related to the problem for the velocity autocorrelation function: strictly speaking, the mean square displacement is only a straight line after the velocity autocorrelation function has become zero. By omitting more and more points of the mean square displacement in the least-squares fit beginning at small times, one can find a value of $s_{\ell}$ beyond which the slope does not vary outside the statistical accuracy. This value of $s_{\ell}$ has to coincide with the time above which the velocity autocorrelation function is zero within the limits of accuracy. So one has two different, though not independent, criteria for the determination of $\ell_{\max }$. 
3.5. Mean free time and distribution of free times

Most of the experimental mean free times have been computed with (2.64) and the standard deviations have been obtained from the differences with the mean value. In a few test runs the validity of the alternative average (2.65) has been checked. Both types of results have been compared with the Boltzmann mean free time.

Also most of the average distributions of free times have been obtained using the straightforward average (2.66), which reads more explicitly

$$
\mathrm{q}_{\mathrm{e}}\left(\mathrm{s}_{\mathrm{k}}\right)=\mathrm{N}_{\mathrm{c}}^{-1} \underset{\sum_{\mathrm{C}}}{\mathrm{N}_{\mathrm{C}}} \mathrm{N}_{\mathrm{T}}^{-1}(\alpha) \sum_{\ell=1}^{\mathrm{N}_{\mathrm{T}}(\alpha)}\left\{\mathrm{H}\left(\mathrm{s}_{\ell}-\mathrm{s}_{\mathrm{k}-1}\right)-\mathrm{H}\left(\mathrm{s}_{\ell}-\mathrm{s}_{\mathrm{k}}\right)\right\} / \mathrm{h},
$$

where $s_{\ell}$ is the reduced free time $\left(t_{\ell} / \tau\right)$ before the $\ell^{t h}$ collision. H denotes the Heaviside function, $\mathrm{H}(\mathrm{s})=0$ for $\mathrm{s}<0$ and $\mathrm{H}(\mathrm{s})=1$ for $\mathrm{s} \geqslant 0$, $\mathrm{k}$ runs from 1 to a maximum value $\mathrm{k}_{\max }$ and the time interval of the histogram is $\mathrm{h}=\mathrm{s}_{\mathrm{k}}-\mathrm{s}_{\mathrm{k}-1}$. The value $\mathrm{h}=0.05$, almost always used, is small enough for a detailed sampling of the distribution and is large enough with respect to statistics. The value $\mathrm{k}_{\max }=400$ was almost always large enough to cover the range of the computed free times. The alternative average (2.67) has been considered as well in some cases, reading

$$
q_{e}^{\prime}\left(s_{k}\right)=\left(\tau_{e}^{\prime / T}\right) N_{C}^{-1} \sum_{\alpha=1}^{N_{C}} \sum_{l}^{N_{T}(\alpha)}\left\{H\left(s_{\ell}-s_{k-1}\right)-H\left(s_{\ell}-s_{k}\right)\right\} / h .
$$

One way to analyze the obtained histograms is to compare them with the right-hand side of (2.63), which we denote by $g_{N}\left(s_{k}\right)$ and which reads explicitly

$$
Q_{N}\left(s_{k}\right)=\left\{\left(1-s_{k-1} \tau / \tau_{N} N\right)^{N-1}-\left(1-s_{k} \tau / \tau_{N} N\right)^{N-1}\right\} \tau / \tau_{N} h
$$

This gives in the thermodynamic limit, $\mathrm{N} \rightarrow \infty$, 


$$
Q\left(s_{k}\right)=\exp \left(-s_{k}\right)\{\exp (h)-1\} / h .
$$

Another way of studying the distribution of free times is to introduce an adjustableparameter $n$, the inverse of the mean free time, in $\Omega_{N}\left(s_{k}\right)$ or $Q\left(s_{k}\right)$ which can take into account that the mean free time deviates from $\tau_{N}$ or $\tau$. The functions

$$
\mathrm{Q}_{\mathrm{N}}\left(\mathrm{s}_{\mathrm{k}} ; \eta\right)=\eta\left\{\left(1-n s_{\mathrm{k}-1} / \mathrm{N}\right)^{\mathrm{N}-1}-\left(1-n s_{\mathrm{k}} / \mathrm{N}\right)^{\mathrm{N}-1}\right\} / \mathrm{h}
$$

and, in the limit $N \rightarrow \infty$,

$$
Q\left(s_{k} ; \eta\right)=n \exp \left(-n s_{k}\right)\{\exp (n h)-1\} / h
$$

are obvious generalizations of (3.12) and (3.13) respectively. As mentioned at the end of section $2.3 \mathrm{q}_{\mathrm{e}}^{\prime}\left(\mathrm{s}_{\mathrm{k}}\right)$ differs from the lefthand side of eq. (2.63) by a factor $\tau_{e}^{\prime}$. This difference can be easily accounted for by absorbing this factor in (3.14) or (3.15), giving after cancellation against the factor $n$ the functions

$$
\hat{\mathrm{Q}}_{\mathrm{N}}\left(\mathrm{s}_{\mathrm{k}} ; \eta\right)=\left\{\left(1-n \mathrm{~s}_{\mathrm{k}-1} / \mathrm{N}\right)^{N-1}-\left(1-n \mathrm{~s}_{\mathrm{k}} / \mathrm{N}\right)^{N-1}\right\} / \mathrm{h}
$$

and

$$
\hat{Q}\left(s_{k} ; \eta\right)=\exp \left(-\eta s_{k}\right)\{\exp (\eta h)-1\} / h .
$$

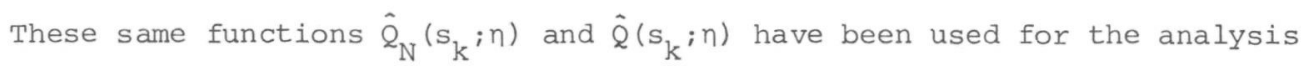
of $q_{e}\left(s_{k}\right)$. Although the choice between (3.14), (3.15) and (3.16), (3.17) is less unique in this case, we have decided to do so, because $q_{e}\left(s_{k}\right)$ and $q_{e}^{\prime}\left(s_{k}\right)$ have the same dimensionality. The results justify this choice. A least-squares fit of $\hat{Q}_{\mathrm{N}}\left(s_{k} ; \eta\right)$ or $\hat{Q}\left(s_{k} ; \eta\right)$ provides us with a value for $\eta_{e}$, which is an estimate for the inverse mean free time. 
References

3.1) System/360 Scientific Subroutine Package IBM Application Program GH2O - 0205 - 4

3.2) Knuth, D.E., The art of computer programming Vol. 2 . Addison-Wesley Publishing Company, Reading (MA) (1969)

3.3) MacLaren, M.D. and Marsaglia, G., J.A.C.M. 12 (1965) 83

3.4) Marsaglia, G., Proc. Nat. Acad. Sci. 61 (1968) 25

3.5) Gruenberger, F. and Mark, A.M., Math. Tables other Aids Comp. $\underline{5}$ (1951) 109

3.6) Amadori, R., J. Comp. Phys. 24 (1977) 450

3.7) Alder, B.J. and Wainwright, T.E., J. Chem. Phys. 31 (1959) 459

3.8) Futrelle, R.P. and McGinty, D.J., Chem. Phys. Letters 12 (1971) 285 
The computations for the two-dimensional Lorentz gas have been performed from very low densities, where deviations from the Boltzmann regime are small, up to high densities, where diffusion becomes impossible. As has been shown in chapter 2 , the effect of finite $N$, the number of scatterers, is larger for lower densities. Therefore, in general the values of $\mathrm{N}$ used for low densities have been chosen larger than those for the higher densities. Since the verification of a series expansion in the density is the main object, the major part of machine time has been spent on the lowdensity region.

In table 4.1 the characteristic parameters and most important results of the molecular-dynamics computations are given. The various columns contain respectively

1) the reduced density $n^{*}$,

2) the number of scatterers $N$,

3) the number of configurations of scatterers $\mathrm{N}_{C^{\prime}}$

4) the total number of correlation sequences $\mathrm{N}_{f} \mathrm{~N}_{c}$ used in the averaging procedure,

5) the experimental mean free time $\tau_{e}$ reduced with respect to the Boltzmann infinite-system mean free time $\tau$,

6) the reciprocal of the collision rate $n_{e}$ as obtained from a least-squares fit of the distribution of free times,

7) the normalized $x^{2}$-variable resulting from the above-mentioned fit,

8) the reduced diffusion coefficient $D_{e} / v \sigma$, as obtained from integrating the velocity autocorrelation function. Up to the density $\mathrm{n}^{*}=0.1$ this function has been computed in 100 equidistant points. 
Table 4.1. Characteristic parameters and results ${ }^{a}$ for the 2-dimensional Lorentz gas,

\begin{tabular}{|c|c|c|c|c|c|c|c|c|c|c|}
\hline $\begin{array}{l}1 \\
n^{*}\end{array}$ & $\begin{array}{l}2 \\
\mathrm{~N}\end{array}$ & $\begin{array}{l}3 \\
\mathrm{~N}_{\mathrm{C}}\end{array}$ & $\begin{array}{c}4 \\
\mathrm{~N}_{\mathrm{f}^{\mathrm{N}}{ }_{\mathrm{C}}} \\
\left(10^{6}\right)\end{array}$ & $\tau e^{5}$ & $\eta_{e}^{-1}$ & $\begin{array}{l}7 \\
x^{2}\end{array}$ & $D_{e}^{8} / v \sigma$ & $\begin{array}{l}9 \\
s_{x}\end{array}$ & $\mathrm{D}_{\mathrm{e}}^{10}$ & $\begin{array}{c}11 \\
\mathrm{~s}_{\mathrm{n}}, \mathrm{s}_{\mathrm{x}}\end{array}$ \\
\hline 0.003 & 8000 & 800 & 20 & $0.9995(3)$ & $0.9994(3)$ & 1.07 & $61.20(9)$ & 15 & & \\
\hline 0.004 & 8000 & 800 & 20 & $0.9993(3)$ & $0.9990(3)$ & 1.15 & $45.69(6)$ & 15 & & \\
\hline 0.004 & 8000 & 4000 & 10 & $0.9999(3)$ & $0.9997(4)$ & 1.15 & $45.68(10)$ & 15 & & \\
\hline 0.005 & 8000 & 800 & 20 & $0.9995(3)$ & $0.9992(3)$ & 1.23 & $36.34(5)$ & 15 & & \\
\hline 0.005 & 2000 & 3200 & 40 & $1.0004(3)$ & $1.0002(3)$ & 1.24 & $36.40(4)$ & 10 & & \\
\hline 0.010 & 8000 & 1600 & 20 & $0.9997(3)$ & $0.9994(3)$ & 1.17 & $17.80(2)$ & 15 & & \\
\hline 0.010 & 2000 & 1600 & 20 & $0.9999(4)$ & $0.9999(3)$ & 0.95 & $17.77(3)$ & 10 & & \\
\hline 0.015 & 8000 & 800 & 10 & $0.9995(4)$ & $0.9991(4)$ & 1.19 & $11.59(2)$ & 15 & & \\
\hline 0.015 & 2000 & 1600 & 20 & $1.0001(4)$ & $1.0000(3)$ & 0.95 & $11.57(2)$ & 10 & & \\
\hline 0.020 & 8000 & 1600 & 20 & $0.9995(4)$ & $0.9992(3)$ & 1.01 & $8.525(13)$ & 15 & & \\
\hline 0.020 & 2000 & 1600 & 20 & $1.0002(4)$ & $1.0000(3)$ & 1.07 & $8.547(13)$ & 10 & & \\
\hline 0.030 & 2000 & 400 & 5 & $1.0003(5)$ & $0.9998(6)$ & 1.02 & $5.498(16)$ & 15 & & \\
\hline 0.040 & 2000 & 400 & 5 & $0.9983(7)$ & $0.9975(5)$ & 0.92 & $3.931(11)$ & 15 & & \\
\hline 0.050 & 2000 & 280 & 3.5 & $1.0005(11)$ & $0.9998(7)$ & 1.27 & $3.049(10)$ & 15 & & \\
\hline 0.050 & 2000 & 2000 & 1 & $1.0018(12)$ & $1.0003(10)$ & 1.09 & $3.039(16)$ & 20 & $3.014(28)$ & 20,100 \\
\hline 0.050 & \multicolumn{3}{|c|}{ alternative } & $0.9985(11)$ & $0.9973(10)$ & 1.05 & & & & \\
\hline 0.060 & 2000 & 400 & 2 & $0.999(1)$ & $0.998(1)$ & 1.02 & $2.424(13)$ & 20 & & \\
\hline 0.070 & 2000 & 320 & 1.6 & $0.999(1)$ & $0.998(1)$ & 1.15 & $2.033(12)$ & 20 & & \\
\hline 0.080 & 2000 & 240 & 1.2 & $0.998(2)$ & $0.998(1)$ & 0.97 & $1.692(10)$ & 20 & & \\
\hline 0.090 & 2000 & 160 & 0.8 & $0.997(2)$ & $0.995(1)$ & 0.98 & $1.442(11)$ & 20 & & \\
\hline 0.100 & 2000 & 160 & 0.8 & $0.998(2)$ & $0.996(1)$ & 1.01 & $1.255(11)$ & 20 & & \\
\hline
\end{tabular}


Table 4.1. (continued).

\begin{tabular}{|c|c|c|c|c|c|c|c|c|c|c|}
\hline $\begin{array}{l}1 \\
n^{*}\end{array}$ & $\begin{array}{l}2 \\
\mathrm{~N}\end{array}$ & $\begin{array}{l}3 \\
\mathrm{~N}_{\mathrm{C}}\end{array}$ & $\begin{array}{c}4 \\
\mathrm{~N}_{\mathrm{f}^{\mathrm{C}} \mathrm{N}_{\mathrm{C}}} \\
\left(10^{6}\right)\end{array}$ & $\begin{array}{c}5 \\
\tau e^{/ \tau}\end{array}$ & $\begin{array}{c}6 \\
n_{e}^{-1}\end{array}$ & $\begin{array}{l}7 \\
x^{2}\end{array}$ & $\begin{array}{c}8 \\
D_{e} / v \sigma\end{array}$ & $\begin{array}{l}9 \\
s_{x}\end{array}$ & $\begin{array}{c}10 \\
D_{e} / v \sigma\end{array}$ & $\begin{array}{c}11 \\
s_{n}, s_{x}\end{array}$ \\
\hline 0.10 & 500 & 200 & 0.5 & $0.999(3)$ & $0.994(2)$ & 1.23 & $1.217(22)$ & 30 & $1.207(21)$ & 30,50 \\
\hline 0.12 & 500 & 200 & 0.5 & $1.004(4)$ & $1.000(2)$ & 1.08 & $0.934(20)$ & 30 & $0.918(28)$ & 30,50 \\
\hline 0.14 & 500 & 200 & 0.5 & $1.002(4)$ & $0.994(2)$ & 1.23 & $0.717(14)$ & 30 & $0.702(16)$ & 30,50 \\
\hline 0.16 & 500 & 200 & 0.5 & $1.002(7)$ & $0.996(2)$ & 1.09 & $0.600(11)$ & 30 & $0.610(12)$ & 30,50 \\
\hline 0.18 & 500 & 200 & 0.5 & $1.007(7)$ & $0.997(3)$ & 1.42 & $0.458(7)$ & 30 & $0.462(12)$ & 30,50 \\
\hline 0.20 & 2000 & 2000 & 1 & $1.021(3)$ & $1.016(1)$ & 1.67 & $0.385(8)$ & 30 & $0.368(5)$ & 30,100 \\
\hline 0.20 & \multicolumn{3}{|c|}{ alternative } & $0.994(6)$ & 0.995 (1) & 1.02 & & & & \\
\hline 0.20 & 500 & 200 & 0.5 & $1.014(5)$ & $1.002(3)$ & 2.04 & $0.364(8)$ & 30 & $0.365(9)$ & 30,50 \\
\hline 0.22 & 500 & 200 & 0.5 & $1.014(11)$ & $1.003(3)$ & 1.21 & $0.308(8)$ & 45 & $0.304(8)$ & 45,50 \\
\hline 0.24 & 500 & 200 & 0.5 & $1.025(8)$ & $1.014(3)$ & 1.66 & $0.249(6)$ & 45 & $0.248(6)$ & 45,50 \\
\hline 0.26 & 500 & 200 & 0.5 & $1.029(10)$ & $1.018(3)$ & 1.22 & $0.206(6)$ & 45 & $0.207(7)$ & 45,50 \\
\hline 0.28 & 500 & 200 & 0.5 & $1.055(12)$ & $1.036(3)$ & 1.73 & $0.153(3)$ & 45 & $0.156(5)$ & 45,50 \\
\hline 0.30 & 500 & 200 & 0.5 & $1.066(9)$ & $1.046(4)$ & 2.82 & $0.139(5)$ & 45 & $0.137(5)$ & 45,50 \\
\hline 0.40 & 2000 & 2000 & 1 & $1.115(5)$ & $1.089(4)$ & 11 & $0.037(3)$ & 90 & $0.040(2)$ & 90,100 \\
\hline 0.40 & \multicolumn{3}{|c|}{ alternative } & $1.018(9)$ & $1.005(2)$ & 1.64 & & & & \\
\hline 0.60 & 2000 & 2000 & 1 & $1.190(9)$ & $1.148(5)$ & 16 & & & & \\
\hline 0.60 & \multicolumn{3}{|c|}{ alternative } & $1.000(10)$ & $0.989(2)$ & 1.41 & & & & \\
\hline 0.80 & 2000 & 2000 & 1 & $1.283(13)$ & $1.211(6)$ & 21 & & & & \\
\hline 0.80 & \multicolumn{3}{|c|}{ alternative } & $1.011(17)$ & $1.004(2)$ & 1.17 & & & & \\
\hline
\end{tabular}

$a_{\text {Notation: }} 0.9995(3)=0.9995 \pm 0.0003 ; 1.283(13)=1.283 \pm 0.013$. 
Time increments of $h=0.1,0.15$ or 0.2 have been used. For densities higher than $n^{*}=0.1$, in addition to the 100 points with $\mathrm{h}=0.1$ or 0.2 the velocity autocorrelation function has been computed in 80 points with a 5 times larger time increment: $\mathrm{h}=0.5$ or 1.0 ,

9) the maximum time $s_{x}$ up to where the velocity autocorrelation function has been integrated,

10) the reduced diffusion coefficient $D_{e} / v \sigma$, as obtained from the slope of the mean square displacement,

11) the minimum time $s_{n}$ and the maximum time $s_{x}$ between which the slope has been determined.

For the lines marked by the word 'alternative' in the second column, the same configurations of scatterers and trajectories of the moving particle have been used as in the preceding line to compute the alternative averages, $\tau_{e}^{\prime}(2.65)$ and $q_{e}^{\prime}\left(s_{k}\right)(3.11)$, instead of the straight-forward averages.

For low densities up to a density $n^{*}=0.2$ it is found that $\tau e^{/ \tau}$ is in good agreement with the value 1 , taking into account the standard deviation. (Reducing $\tau_{e}$ with respect to $\tau_{N}$ instead of $\tau$ gives a negligible change in the numbers). For higher densities $\tau_{e} / \tau$ becomes significantly too high. For the few cases studied at high density, $\tau_{e}^{\prime / \tau}$ does appear to be in agreement with the theoretical value 1.

For the case of the Ehrenfest wind-tree model, wood ${ }^{4.1)}$ has also observed that $\tau_{e}$ is a good approximation to $\tau_{e}^{\prime}$ for low densities and a bad one for high densities. His explanation for this effect is that for higher densities the distribution of mean free times, as obtained for different configurations of scatterers, becomes broader. This leads to larger values for the higher relative moments of the distribution and these become non-negligible in an expansion of $\tau_{e}$ in terms of $\tau_{e}^{\prime}$. More explicitly, one can make an expansion of the average of the inverse of variable $t$ in terms of the inverse of the average of $t$ and one obtains 


$$
\begin{aligned}
\left\langle t^{-1}\right\rangle & =\langle t\rangle^{-1}\left\langle\left\{1+\frac{t-\langle t\rangle}{\langle t\rangle}\right\}^{-1}\right\rangle \\
& =\langle t\rangle^{-1}\left\{1+\frac{\left.\langle t-\langle t\rangle\}^{2}\right\rangle}{\langle t\rangle}-\frac{\left.\langle t-\langle t\rangle\}^{3}\right\rangle}{\langle t\rangle\rangle^{2}}+\ldots\right\} .
\end{aligned}
$$

The fitted values $\eta_{e}$ for $n$ have been obtained from a minimization procedure for the reduced $x^{2}$-variable

$$
x^{2}=(1 / 199) \sum_{k=1}^{200}\left\{\hat{Q}\left(s_{k} ; n\right)-q_{e}\left(s_{k}\right)\right\}^{2} / \sigma_{k}^{2},
$$

where $\hat{Q}\left(s_{k} ; \eta\right)$ and $q_{e}\left(s_{k}\right)$ are defined in (3.16) and (3.9) respectively and $\sigma_{k}$ is the standard deviation of $q_{e}\left(s_{k}\right)$. Only the first 200 intervals of the distributions have been used rather than all the 400 computed ones because of the smaller statistical accuracy of the last 200 intervals. The standard deviations of $n_{e}$ have been obtained from the variance-covariance matrix in the fitting procedure.

The inverted values $\eta_{e}^{-1}$ behave in much the same way as $\tau_{e} / \tau$. of course, for the so-called alternative lines in table $4.1 \mathrm{q}_{\mathrm{e}}^{\prime}\left(\mathrm{s}_{\mathrm{k}}\right)$, defined in (3.10), has been used in eq. (4.2) rather than $\mathrm{q}_{e}\left(\mathrm{~s}_{\mathrm{k}}\right)$.

The reduced $x^{2}$-variable gives a measure for the correctness of the fit. For the lower densities the values for $x^{2}$ are in reasonable agreement with the most probable value 1 , be it that they are somewhat high in general. For high densities the deviations from 1 are significant as regards the results for the straightforward average $q_{e}\left(s_{k}\right)$. Again for the alternative average $q_{e}^{\prime}\left(s_{k}\right)$ the results are quite satisfactorily close to 1 . In fig. 4.1 a few examples are given of the behaviour of the terms $x_{k}=\left\{q_{e}\left(s_{k}\right)-\hat{Q}\left(s_{k} ; \eta_{e}\right)\right\} / \sigma_{k}$ and $x_{k}^{\prime}=\left\{q_{e}^{\prime}\left(s_{k}\right)-\hat{Q}\left(s_{k} ; \eta_{e}\right)\right\} / \sigma_{k}$ occurring in the sum of eq. (4.2). Only the first 120 values of $k$ are shown, giving a range for the reduced time $s_{k}$ from 0 to 6 .

As regards the alternative average $x_{k}^{\prime}$; the major part of the values is situated between +1 and -1 as expected. For a low density, $n^{*}=0.05$, this also holds for the straightforward average $x_{k}$. For a high density, $\mathrm{n}^{*}=0.8$, large deviations from the expected behaviour are observed. 


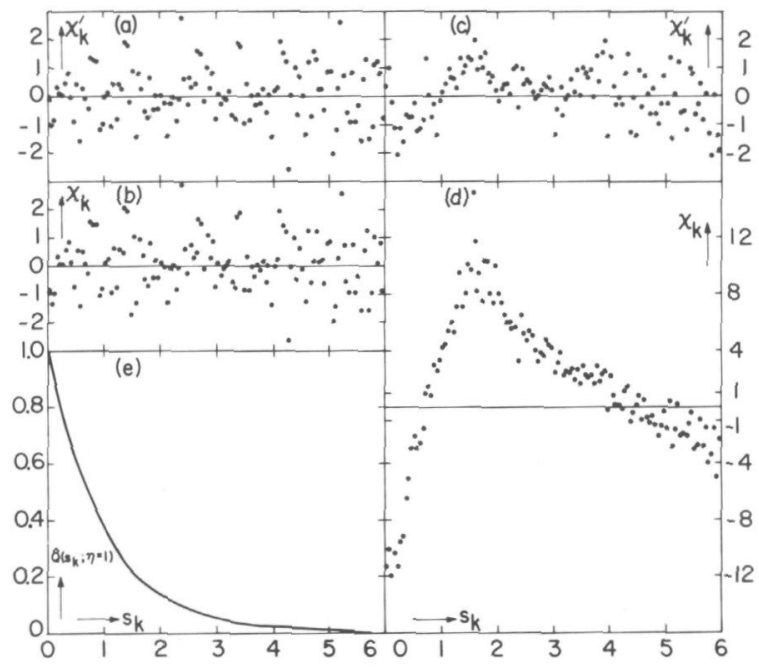

Fig. 4.1. Details of the 2-dimensional free-times distribution. For the lowdensity case $n^{*}=0.05\left(N=2000, N_{c}=2000\right) x_{k}^{\prime}$ is given in (a) and $x_{k}$ in (b). For the high-density case $r^{*}=0.8 x_{k}^{\prime}$ is given in (c) and $x_{k}$ in (d). In (e) $\hat{Q}\left(s_{k} ; n=1\right)$ is given for reference to an absolute scale. The abscissa is identical in all cases.

Qualitatively these deviations are in agreement with the differences between the free-times distributions of the first and the second free time, shown in fig. A.6.

The diffusion coefficients obtained from the velocity autocorrelation function agree reasonably well with those from the mean square displacement, as should be. For further analysis the former ones have been used. In case more than one value is mentioned in column 8 of table 4.1 at the same density, the average has been determined using weight factors proportional to the inverse square of the pertinent standard deviations.

In fig. 4.2 the inverted experimental diffusion coefficients are given as a function of the density. Error bars have been omitted when they are smaller that the dots. For low densities (up to $\mathrm{n}^{*}=0.1$ ) the agreement with the first few terms of the density expansion (2.25) is very good. 

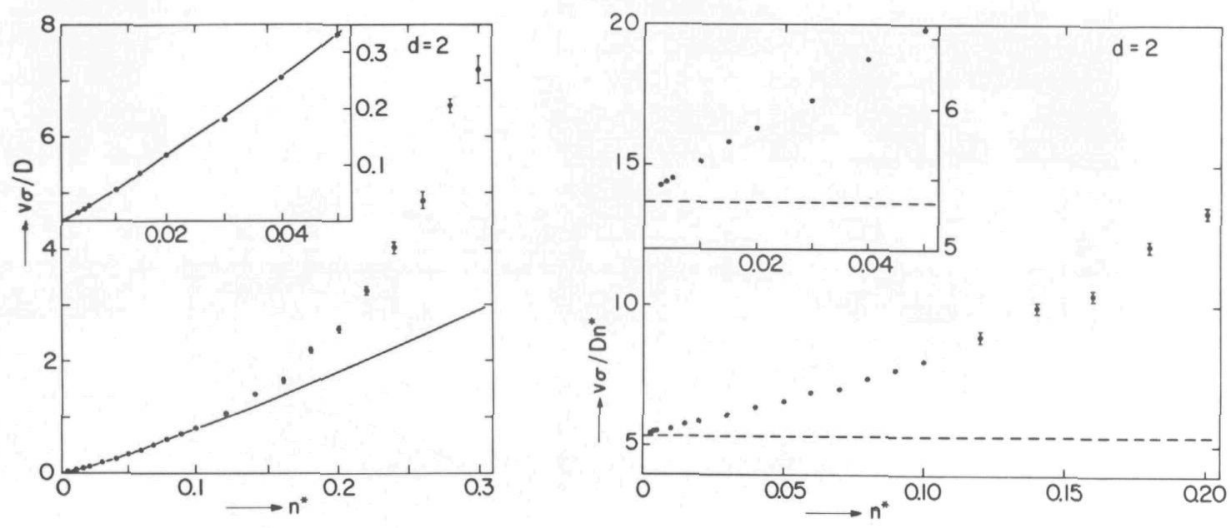

Fig. 4.2. The experimental inverse diffusion coefficient as a function of the density for the 2-dimensional case (dots). The sum of the first four terms in the density expansion, $v \sigma / D=c_{1} n^{*}+$ $c_{2}^{\prime} n^{*^{2}} \operatorname{In} n^{*}+c_{2} n^{*^{2}}+c_{3}^{\prime \prime} n^{*^{3}}\left(\operatorname{In} n^{*}\right)^{2}$, is presented by the line. For low densities the data are shown on an entarged scale.

Fig. 4.3. Comparison of $v \sigma / D e^{n *}$ (dots) with the Boltzmann term $c_{1}=16 / 3$ (dashed Iine).

For a closer inspection of the low-density region, $\mathrm{v} \sigma / \mathrm{D}_{\mathrm{e}}$ is divided by $\mathrm{n}^{*}$ and the result is compared in fig. 4.3 with the Boltzmann value $c_{1}$ (dashed line). For decreasing densities, $v \sigma / D_{e} n^{*}$ converges rather well towards $c_{1}$, which is the leading contribution for low densities.

Next, the Boltzmann contribution $c_{1}$ is subtracted from $v \sigma / D_{e} n^{*}$ and the difference is again diviced by the density. The resulting data show a remarkable increase for $\mathrm{n}^{*} \rightarrow 0$ in fig. 4.4. The seven lowest densities for which the most extensive computations have been carried out show clearly the decrease of the first logarithmic term for increasing density. They indicate that the coefficient $c_{2}^{\prime}$ is of the right order of magnitude. From these data it can be concluded that the density expansion of the inverted diffusion coefficient for the 2-dimensional Lorentz gas does contain a logarithmic term.

This conclusion is also suggested by a comparison of the least-squares fits of either $\alpha_{0} \ln n^{*}+\alpha_{1}+\alpha_{2} n^{*}\left(\ln n^{*}\right)^{2}$ or $a_{0}+a_{1} n^{*}+a_{2} n^{* 2}$ to the 

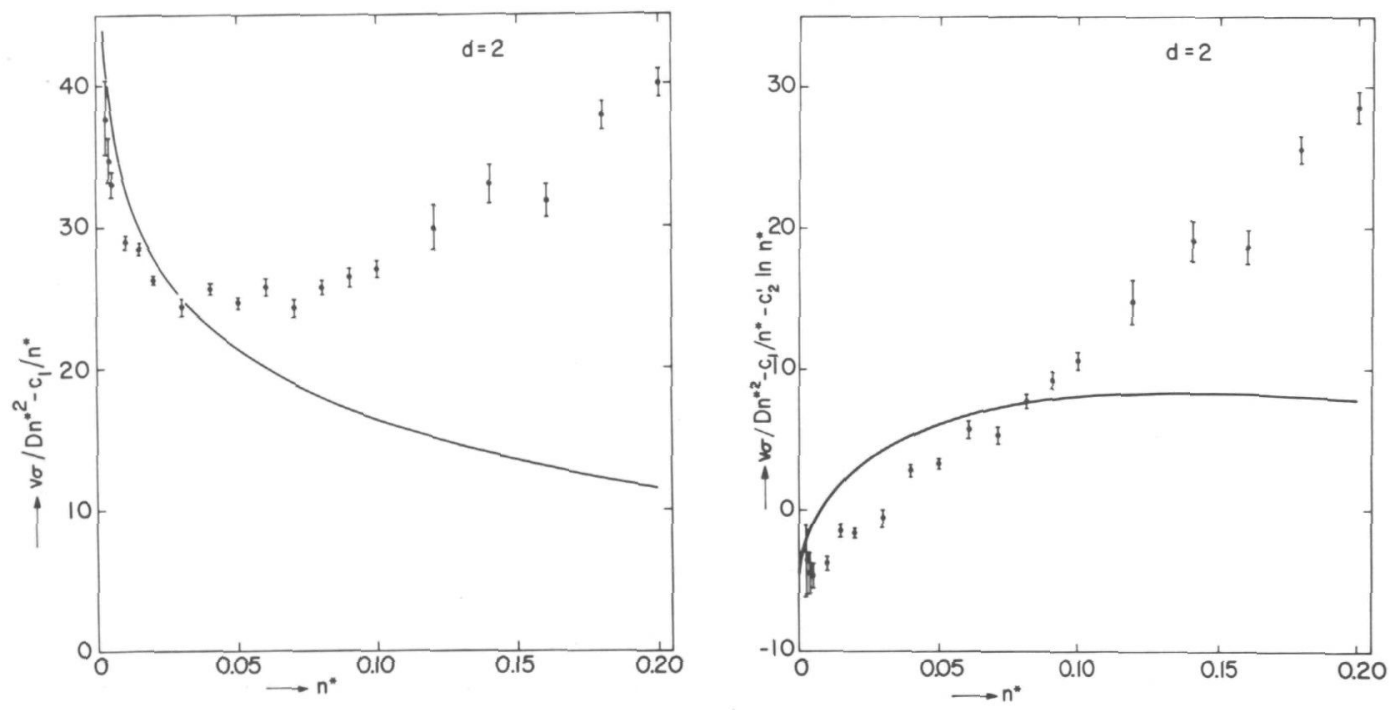

Fig. 4.4. Comparison of $\left(v_{\sigma} / D e^{n^{*}}-c_{1}\right) / n^{*}$ (dots) with the most important Logarithmic term $c_{2}^{\prime}$ In $n^{*}$ (Iine).

Fig. 4.5. Comparison of $\left(v \sigma / D e^{n^{*}}-c_{1}\right) / n^{*}-c_{2}^{\prime} \ln n^{*}$ (dots) with the remaining terms $c_{2}+c_{3}^{\prime \prime} n^{*}\left(\ln n^{*}\right)^{2}$ ( (ine).

experimental data $\left(v \sigma / D_{e}-C_{1} n^{*}\right) / n^{*}$. This has been done starting from low densities with an increasing number of data points, as given in fig. 4.4. The results for the coefficients are tabulated in table 4.2 for a few cases. Quantitatively, these results are not very meaningful, both because of the large errors for the coefficients and hence their fluctuating values and because of large values for the $x^{2}$-variable, leading to values of about $90 \%$ in the $x^{2}$ - test (this should be $50 \%$ ). The important point that this comparison does show is that in case one wants to describe the data with a power series, one needs coefficients increasing by about a factor of 10 with each order.

A more appropriate numerical test on the validity of the series expansion (2.25) is provided by the difference of the experimental inverted diffusion coefficient and the predicted one, divided by the experimental standard deviation: $x_{n^{*}}=\left(v \sigma / D_{e}-v \sigma / D\right) / \sigma_{D}\left(v \sigma / D_{e}\right)^{2}$, where 
Table 4.2. Least-squares fit results of either $\alpha_{0} \ln n^{*}+\alpha_{1}+\alpha_{2} n^{*}\left(\ln n^{*}\right)^{2}$ or $a_{0}+a_{1} n^{*}+a_{2} n^{*^{2}}$ to a number $i_{\max }$ of data points as given in fig. 4.4 starting from low densities. The errors given have been obtained from the variance-covariance matrix in the fitting procedure.

\begin{tabular}{|c|c|c|c|c|c|c|}
\hline$i_{\max }$ & $\alpha_{0}$ & $\alpha_{1}$ & $\alpha_{2}$ & $a_{0}$ & $\begin{array}{c}a_{1} \\
\left(10^{1}\right)\end{array}$ & $\begin{array}{c}a_{2} \\
\left(10^{2}\right)\end{array}$ \\
\hline 6 & $-7(7)$ & $-9(44)$ & $21(6)$ & $37(2)$ & $-85(42)$ & $164(153)$ \\
7 & $-6(5)$ & $0.5(29)$ & $9(36)$ & $36(1)$ & $-70(19)$ & $104(51)$ \\
8 & $-13(5)$ & $-44(30)$ & $67(36)$ & $36(3)$ & $-74(11)$ & $120(22)$ \\
9 & $-13(4)$ & $-47(24)$ & $71(28)$ & $34(2)$ & $-53(12)$ & $68(20)$ \\
10 & $-14(4)$ & $-53(26)$ & $79(30)$ & $34(1)$ & $-47(10)$ & $58(14)$ \\
\hline
\end{tabular}

$\sigma_{D}$ is the standard deviation in $D_{e} / v \sigma$. The absolute value of $x_{n}$ should be of order 1 and the + and - signs should be distributed at random. From the second column of table 4.3 it is clear that $x_{n^{*}}$ fails the test for the values of the coefficients given in (2.26), even for low densities. The values are all negative and their amplitude is much too large. The deviations for higher densities are not so serious, because they can be accounted for by higher order terms in the series expansion.

In fig. 4.5 the discrepancy is also illustrated, by plotting $\left(v \sigma / D_{e}-c_{1} n^{*}-c_{2}^{\prime} n^{2} l n n^{*}\right) / n^{*}$ and comparing it with $c_{2}+c_{3}^{\prime \prime} n^{*}\left(l n n^{*}\right)^{2}$. From this figure one might prefer a larger negative value for $\mathrm{c}_{2}$, say $c_{2}=-8.5$, than the theoretically calculated one $\left(c_{2}=-4.68\right)$. From column 3 of table 4.3 it is seen that this gives a more satisfactory behaviour for $x_{n^{*}}$ for the lowest densities. This might justify a reexamination of the diagrams contributing to $\mathrm{c}_{2}$ and their computation.

For the velocity autocorrelation function at low densities, some examples are given in fig. 4.6 for densities up to $n^{*}=0.02$. These functions deviate by a small amount from the Boltzmann result $\phi_{B}(s)$ [ see (2.45)], therefore the difference $\phi(s)-\phi_{B}(s)$ is shown. In representing the data points by lines no smoothing was involved. For times 
Table 4.3. Comparison of the experimental diffusion coefficient vo/D $D_{e}$ ith the series expansion (2.25): $x_{n *}=\left(v \sigma / D_{e}-v \sigma / D\right) / \sigma_{D}\left(v \sigma / D_{e}\right)^{2}$, where $\sigma_{D}$ is the standard deviation in $D_{e} / v \sigma$. In column 2 the coefficients in vo/D have been chosen according to (2.26). In column 3 the value $c_{2}=-8.5$ has been used rather thon -4.68 .

\begin{tabular}{|c|c|c|}
\hline$n^{*}$ & $x_{n}$ & $x_{n}$ \\
& $c_{2}=-4.68$ & $c_{2}=-8.5$ \\
\hline 0.003 & -0.55 & 0.95 \\
0.004 & -1.79 & 0.66 \\
0.005 & -3.87 & 0.49 \\
0.010 & -7.95 & -0.84 \\
0.015 & -7.99 & 1.61 \\
0.020 & -13.94 & -1.57 \\
0.030 & -8.21 & -1.72 \\
0.040 & -5.71 & 2.88 \\
0.050 & -7.90 & 2.91 \\
0.060 & -1.73 & 4.48 \\
0.070 & -3.32 & 3.13 \\
0.080 & 0.18 & 7.18 \\
0.090 & 2.05 & 7.90 \\
0.100 & 3.95 & 9.86 \\
\hline
\end{tabular}

$s>6$ an enlarged scale is used to give an idea of the influence of the cutoff at $s=10$ or $s=15$ on the values of $D_{e} / v \sigma$ listed in column 8 of table 4.1. For a number of points, error bars are given. It should be noted that for times of the order $s=10$ no substantial contribution to the diffusion coefficient can be expected for the densities shown.

For the investigation of the asymptotic behaviour for long times the function $\phi(s)-\phi_{B}(s)$ has also been used. For low densities the contribution due to the ring diagrams is dominant. From these diagrams the asymptotic expression $\phi_{A}(s)$ has been derived [see (2.46)]. To improve thestatistics, 


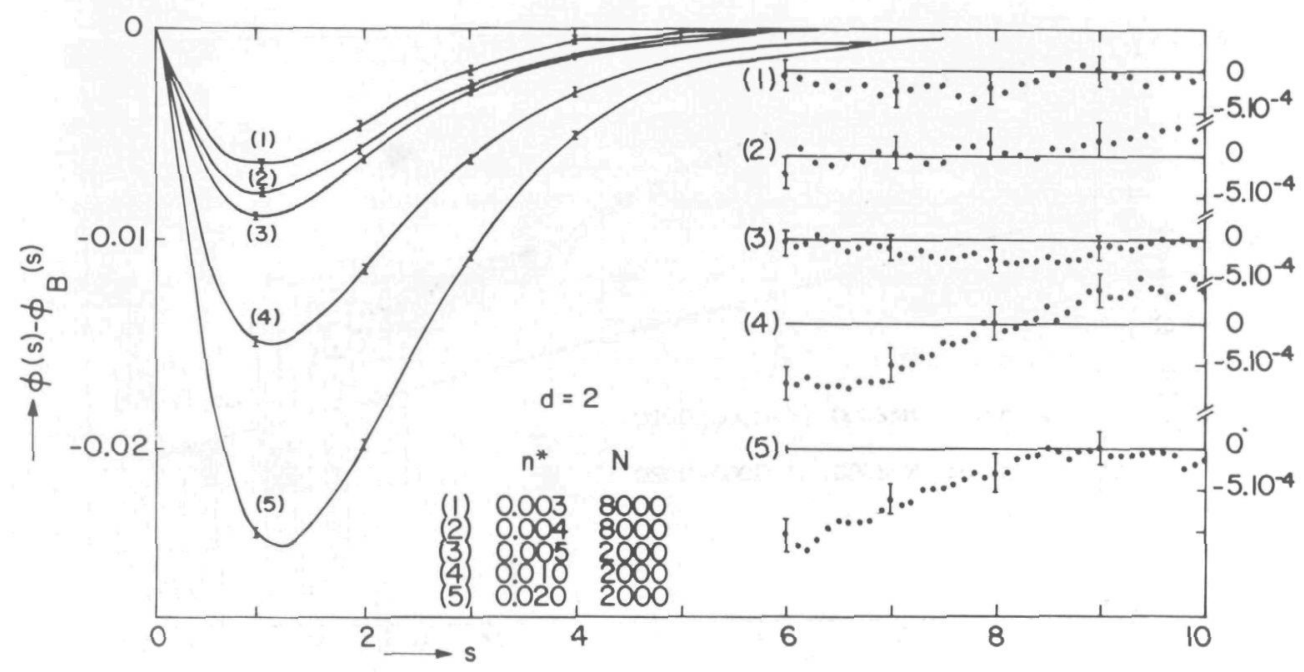

Fig. 4.6. The difference of the experimental velocity autocorrelation function $\phi(s)$ and the Boltzmann result $\phi_{B}(s)$ for five of the Low densities (lines). In drowing the lines no smoothing was involved. The tail for $s>6$ has been given on a four times larger scale (dots).

averages over the lowest-densities results up to $n^{*}=0.02$ have been taken, including a weight factor $1 / \mathrm{n}^{*}$ ( ince $\phi_{A}(s)$ is linear in the density). Two averages have been computed this way: one for results with time increment $h=0.1$ [dots in fig 4.7] and the other one with $h=0.15$ [crosses]. The resulting data are compared in fig 4.7 with $\phi_{A}(s) / n^{*}$ on a $\log -\log$ scale. From the comparison one can state that for times $\mathrm{s}<9$ the asymptotic behaviour as predicted by Ernst and Weyland has not yet set in. For larger times the data do not disagree with the asymptotic expression, but in this region the values are too small compared to the error bars to allow for a more specific conclusion. The time integral over $\phi_{A}(s)$. for large $s$ is finite, in contrast to the moving-hard-disks case studied by Alder and Wainwright ${ }^{4.2)}$, and would contribute only a fraction to the diffusion coefficient for $s>10$ of $3 \times 10^{-9}$ for $n^{*}=0.005$ and of $6 \times 10^{-7}$ for $n^{*}=0.02$. 


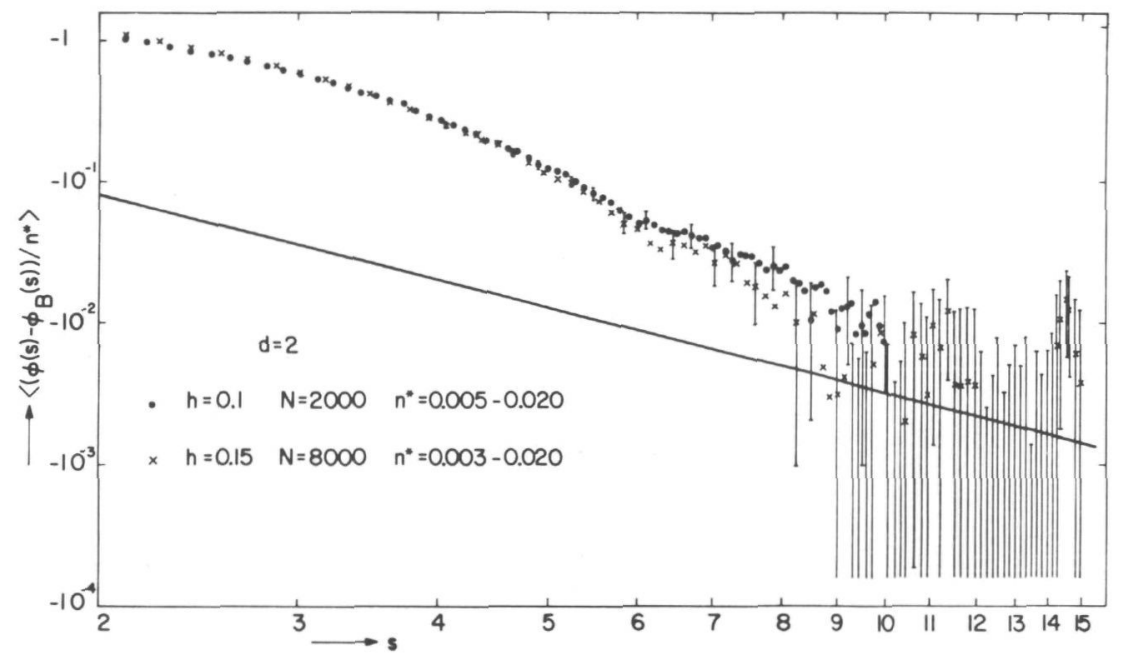

Fig. 4.7. The function $\phi(s)-\phi_{B}(s)$, averaged over several densities with weight factor $1 / n^{*}$, plotted on a log-log scale. The dots represent the average for $h=0.1, N=2000$ and the crosses the one for $h=0.15, N=8000$. The straight line represents the corresponding asymptotic expression $\phi_{A}(s) / n^{*}=-1 /\left(\pi s^{2}\right)$.

For higher densities $\phi(s)$ is significantly different from zero even for $s>10$. Therefore, $\phi(s)$ has been calculated for larger values of $s$ as well. In fig $4.8 \phi(s)$ is shown for 3 densities and the tails are shown on an enlarged scale. In fig. 4.9 the corresponding mean square displacement $\Delta(s)$ is shown. It has been multiplied by $\mathrm{n}^{*^{2}}$ to arrive at a suitable scale: one factor $\mathrm{n}^{*}$ for the reduction of the time axis by $\tau$ and a second factor $\mathrm{n}^{*}$ for the linear term in the series expansion of $\mathrm{vo/D}$. It is clear that for higher densities one has to wait longer before $\Delta(s)$ becomes linear. For $n^{*}=0.3$ it is doubtful whether it becomes linear at all. The explanation is that at high densities it becomes very likely that scatterers in a configuration form more or less closed pockets from which the moving particle cannot escape or at least not within a reasonable short time. It has not been possible to determine the percolation limit, i.e. a critical density $\mathrm{n}_{\mathrm{C}}^{*}$, above which no diffusion is possible anymore. 

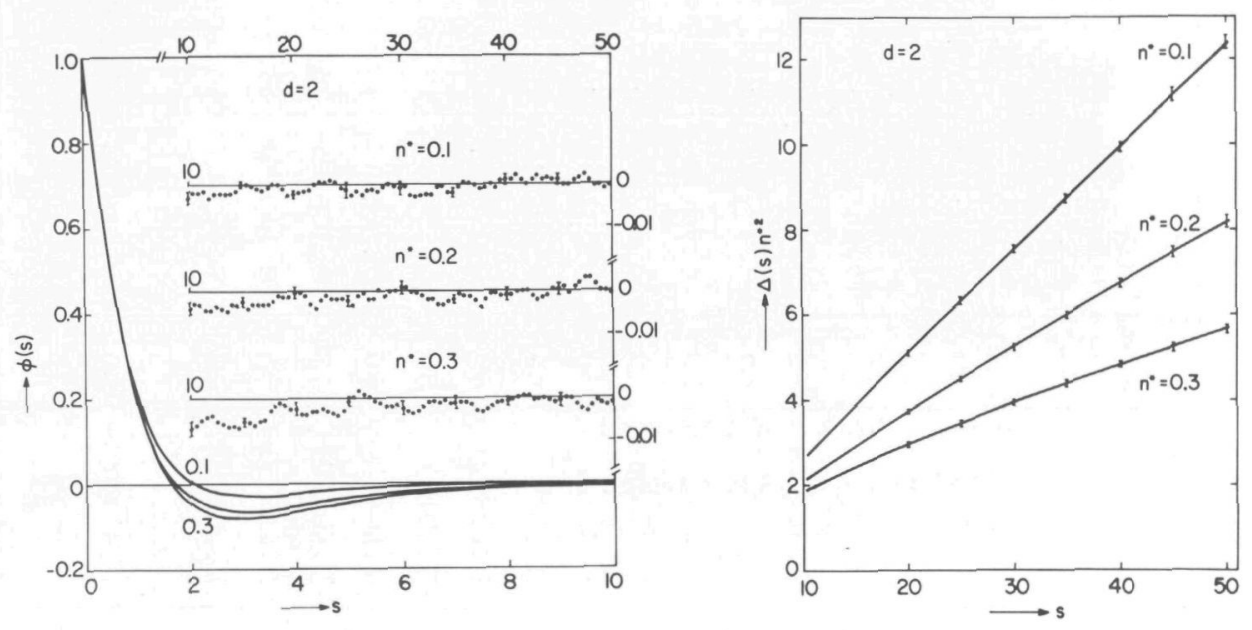

Fig. 4.8. The velocity autocorrelation function $\phi(s)$ for $s<10$ has the smallest negative value for $n^{*}=0.1$ and the largest one for $n^{*}=0.3$ (Zines). The statistical error is slightly Zarger than the line thickness. The tail for $10<s<50$ is given for the same three densities on an enlarged scale (dots).

Fig. 4.9. The reduced mean square displacement $\Delta(s) n^{*}$ for three densities starting with $s=10$. The linear behaviour sets in for a larger time the higher the density.

\subsection{Three-dimensional Lorentz gas}

The calculations performed for the three-dimensional Lorentz gas are much less extensive than for the two-dimensional case. From (2.27) it can be derived that the evidence for logarithmic terms in the density expansion for the inverse diffusion coefficient is much harder to obtain in three dimensions than in two dimensions. Therefore, having obtained some results (cases with $N=100$ in table 4.4) showing good agreement with the first two terms in the expansion it was decided to spend no more time on the three-dimensional case. Later on some more extensive computations (cases with $N=2000$ in table 4.4) have been performed for the study of the non- 
Table 4.4. Characteristic parameters and results for the 3-dimensional Lorentz gas

\begin{tabular}{|c|c|c|c|c|c|c|c|c|c|c|c|}
\hline $\begin{array}{c}1 \\
n^{*}\end{array}$ & 2 & $\begin{array}{l}3 \\
\mathrm{~N}_{\mathrm{C}}\end{array}$ & $\begin{array}{c}4 \\
N_{f^{N}{ }_{C}} \\
\left(10^{6}\right)\end{array}$ & $\begin{array}{c}5 \\
\tau e^{/ \tau}\end{array}$ & $\begin{array}{c}6 \\
\tau_{N} / \tau\end{array}$ & $\begin{array}{c}7 \\
\eta_{e}^{-1}\end{array}$ & $\begin{array}{l}8 \\
x^{2}\end{array}$ & $\begin{array}{c}9 \\
D_{e} / v \sigma\end{array}$ & $\begin{array}{l}10 \\
S_{x}\end{array}$ & $\begin{array}{c}11 \\
D_{e} / v \sigma\end{array}$ & $\begin{array}{c}12 \\
s_{n}, s_{x}\end{array}$ \\
\hline 0.01 & 2000 & 480 & 0.96 & $0.999(1)$ & 1.000 & $0.998(1)$ & 1.19 & $10.55(11)$ & 40 & $10.52(10)$ & 40,100 \\
\hline 0.05 & 2000 & 100 & 1 & $1.001(2)$ & 1.000 & $1.000(1)$ & 1.09 & $1.98(2)$ & 50 & $1.97(2)$ & 50,100 \\
\hline 0.05 & 100 & 100 & 0.15 & $1.004(5)$ & 0.998 & & & $2.02(7)$ & 50 & $2.04(6)$ & 45,50 \\
\hline 0.10 & 2000 & 500 & 1 & 0.998 (1) & 1.000 & $0.998(1)$ & 1.23 & $0.902(8)$ & 60 & $0.895(7)$ & 60,100 \\
\hline 0.10 & 100 & 20 & 0.15 & $0.972(7)$ & 0.996 & & & $0.93(3)$ & 50 & $0.93(3)$ & 45,50 \\
\hline 0.15 & 100 & 20 & 0.15 & $0.95(1)$ & 0.994 & & & $0.53(2)$ & 50 & $0.54(2)$ & 45,50 \\
\hline 0.20 & 2000 & 500 & 1 & $1.001(2)$ & 1.000 & $1.000(1)$ & 1.05 & $0.379(4)$ & 60 & $0.377(4)$ & 60,100 \\
\hline 0.20 & 100 & 20 & 0.15 & $1.02(2)$ & 0.992 & & & $0.41(3)$ & 50 & $0.40(3)$ & 45,50 \\
\hline 0.25 & 100 & 20 & 0.15 & $0.97(2)$ & 0.990 & & & $0.28(1)$ & 50 & $0.28(1)$ & 45,50 \\
\hline 0.30 & 2000 & 500 & 1 & $1.003(2)$ & 0.999 & $1.002(1)$ & 1.16 & $0.198(2)$ & 60 & $0.198(2)$ & 60,100 \\
\hline 0.30 & 100 & 20 & 0.15 & $1.02(2)$ & 0.987 & & & $0.20(1)$ & 50 & $0.21(1)$ & 45,50 \\
\hline 0.35 & 100 & 20 & 0.15 & $1.03(3)$ & 0.985 & & & $0.16(1)$ & 50 & $0.16(1)$ & 45,50 \\
\hline 0.40 & 2000 & 500 & 1 & $1.008(3)$ & 0.999 & $1.006(1)$ & 1.07 & $0.108(2)$ & 70 & $0.105(2)$ & 70,100 \\
\hline 0.40 & 100 & 20 & 0.15 & $1.00(3)$ & 0.983 & & & $0.115(5)$ & 50 & $0.115(5)$ & 45,50 \\
\hline 0.60 & 2000 & 2000 & 1 & $1.045(6)$ & 0.999 & $1.040(2)$ & 2.66 & $0.0298(8)$ & 90 & $0.0299(6)$ & 90,100 \\
\hline 0.60 & \multicolumn{3}{|c|}{ alternative } & $0.990(8)$ & 0.999 & $0.991(1)$ & 1.16 & & & & \\
\hline 0.80 & 2000 & 2000 & 1 & $1.095(8)$ & 0.998 & $1.084(2)$ & 4.34 & $0.0102(5)$ & 90 & $0.0101(4)$ & 90,100 \\
\hline 0.80 & \multicolumn{3}{|c|}{ alternative } & $0.998(8)$ & 0.998 & $0.994(1)$ & 1.06 & & & & \\
\hline
\end{tabular}


gaussian behaviour of Van Hove's self correlation function which can be related to the asymptotic behaviour of the velocity autocorrelation function ${ }^{4.3)}$. However the unfavorable ratio between the magnitude of these non-gaussian correlations and the statistical accuracy obtained prevented us from presenting these results here.

In table 4.4 the results for the three-dimensional case are presented. This table has the same structure as table 4.1, except for one column which has been added (column 6 ). The values of the reiuced Boltzmann mean free time for a finite system, $\tau_{N} / \tau$, are given in this column, since the deviation from the infinite-system value is not negligible in all cases.

At low densities, like in the two-dimensional case, $\tau_{e} / \tau$ is in good agreement with the theoretical value 1 , or rather $\tau_{N} / \tau$. For high densities $\tau_{e} / \tau$ is significantly too high, the alternative average $\tau_{e}^{1 / \tau}$ giving satisfactory results. The results on the free-times distribution, characterized by $n_{e}^{-1}$ and $x^{2}$, confirm this behaviour for the cases the distribution has been determined. The fitted value $\eta_{e}^{-1}$ is in good agreement with $\tau_{e} / \tau$ and $x^{2}$ shows only large deviations from 1 for the straightforward average at high densities.

The diffusion coefficients $D_{e} / v \sigma$ shown in column 9 (table 4.4) have been obtained by integrating the velocity autocorrelation function. They are in good agreement with the ones obtained from the mean square displacement given in column 11. For the situations with $\mathrm{N}=100$ the velocity autocorrelation function has been determined in 100 equidistant points with a time increment $h=0.1$ and in addition to these for larger times in 80 points with a time increment $h=0.5$. For the situations with $\mathrm{N}=2000$ both the time increments have been doubled: giving $\mathrm{h}=0.2$ and $\mathrm{h}=1.0$ respectively. It should be noted that the upper bound $\mathrm{s}_{\mathrm{x}}$ (column 10) for $n^{*}=0.6$ and $n^{*}=0.8$ may be too small or even does not exist, which would mean that diffusion is not possible at these high densities.

In fig. 4.10 the inverted experimental diffusion coefficients are given as a function of density; the dots represent results for the $N=2000$ systems and the crosses some of the results for the $N=100$ 


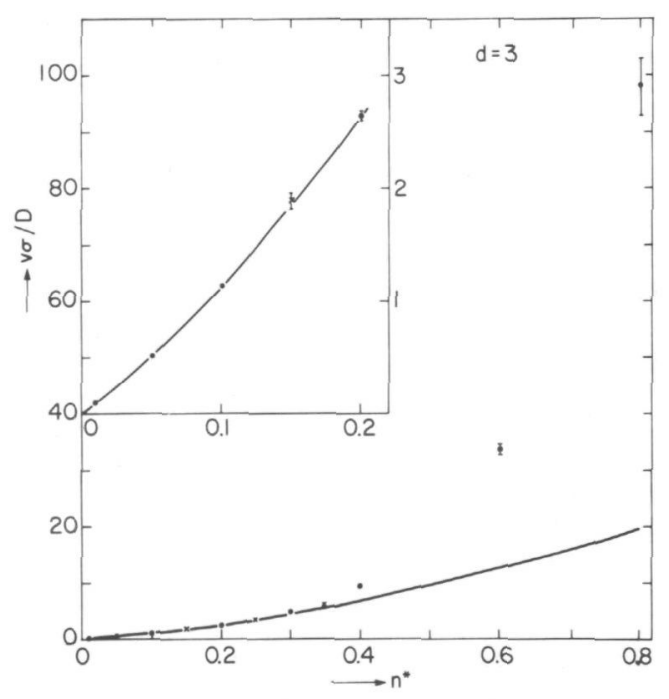

Fig. 4.10. The experimental inverse diffusion coefficient as a function of the density for the 3-dimensional case with $N=2000$ (dots) and $N=100$ (crosses). The sum of the first three terms in the density expansion, $v \sigma / D=b_{1} n^{*}+b_{2} n^{* 2}+b_{3}^{\prime} n^{* 3}$ In $n^{*}$, is presented by the line. For low densities the data are shown on an entarged scale.

systems. Error bars have been omitted when they were smaller than the symbols used. For low densities up to $\mathrm{n}^{*}=0.25$ the agreement with the full line representing the first few terms of the density expansion (2.27) is very good. Dividing $v \sigma / D_{e}$ by $n^{*}$ gives in fig. 4.11 a nice reproduction of the Boltzmann contribution $b_{1}=3 \pi$ (dashed line) going to $n^{*}=0$ and the data agree reasonably with the remaining terms of the series expansion $\mathrm{b}_{2} \mathrm{n}^{*}+\mathrm{b}_{3}^{\prime} \mathrm{n}^{2} \ln \mathrm{n}^{*}($ full line). Substracting the Boltzmann contribution and dividing once more by the density gives the results as shown in fig. 4.12. The full line represents $b_{2}+b_{3}^{\prime} n^{*} l n n^{*}$. The fact that this line hardly deviates from a constant illustrates the smallness of the logarithmic term. The agreement between the data at low densities and the remaining part of the series expansion is not so good on this scale. Although the 

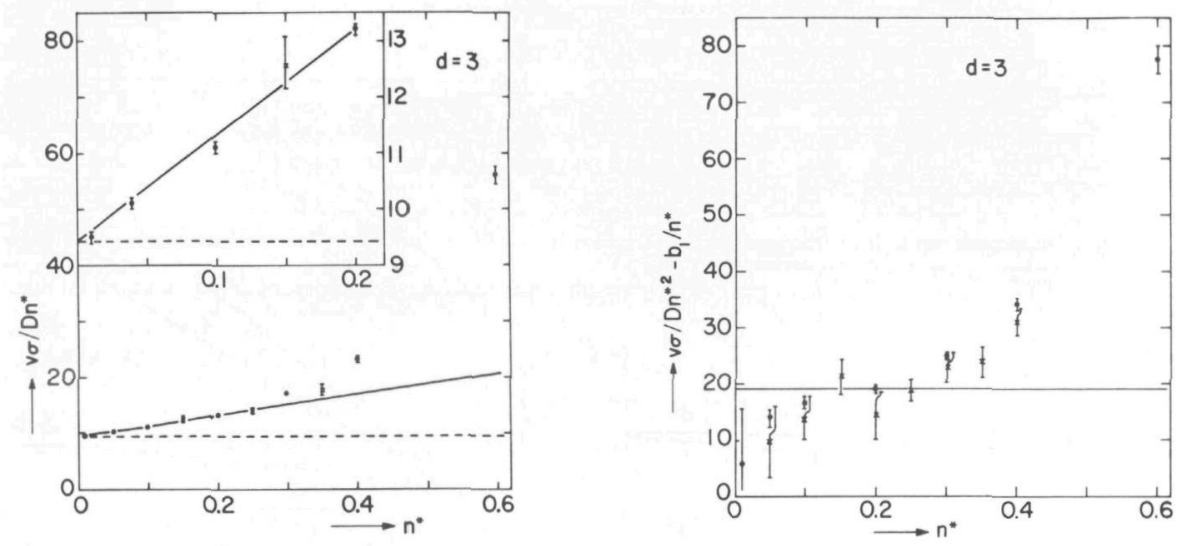

Fig. 4.11. Comparison of vo/D $e^{n^{*}}[\operatorname{dots}(N=2000)$ and crosses $(N=100)]$ with the Boltzmann term $b_{1}=3 \pi$ (dashed line) and with $b_{1}+b_{2} n^{*}+b_{3}^{\prime} n^{* 2}$ in $n^{*}$ (fuzz zine).

Fig. 4.12. Comparison of $\left(v \sigma / D_{e^{n *}}-b_{1}\right) / n^{*}[$ dots $(N=2000)$ and crosses $(N=100)$ ] with the remaining terms $b_{2}+b_{3}^{\prime} n^{*} \ln n^{*}$ (Iine).

error bars are large, the data show preference for a lower value of $\mathrm{b}_{2}$. The velocity autocorrelation function $\phi(s)$ is shown for some densities (situation with $\mathrm{N}=2000$ ) in fig. 4.13, the error bars are smaller than the thickness of the drawn lines. The tails up to 100 mean free times are shown on an enlarged scale (dots). The corresponding mean square displacements are given in fig. 4.14. For every tenth point the error bar is given, whenever it is larger than the thickness of the line drawn through the data. It is seen that for the high density $n^{*}=0.8 \Delta(\mathrm{s})$ does not become linear before $s=100$. 

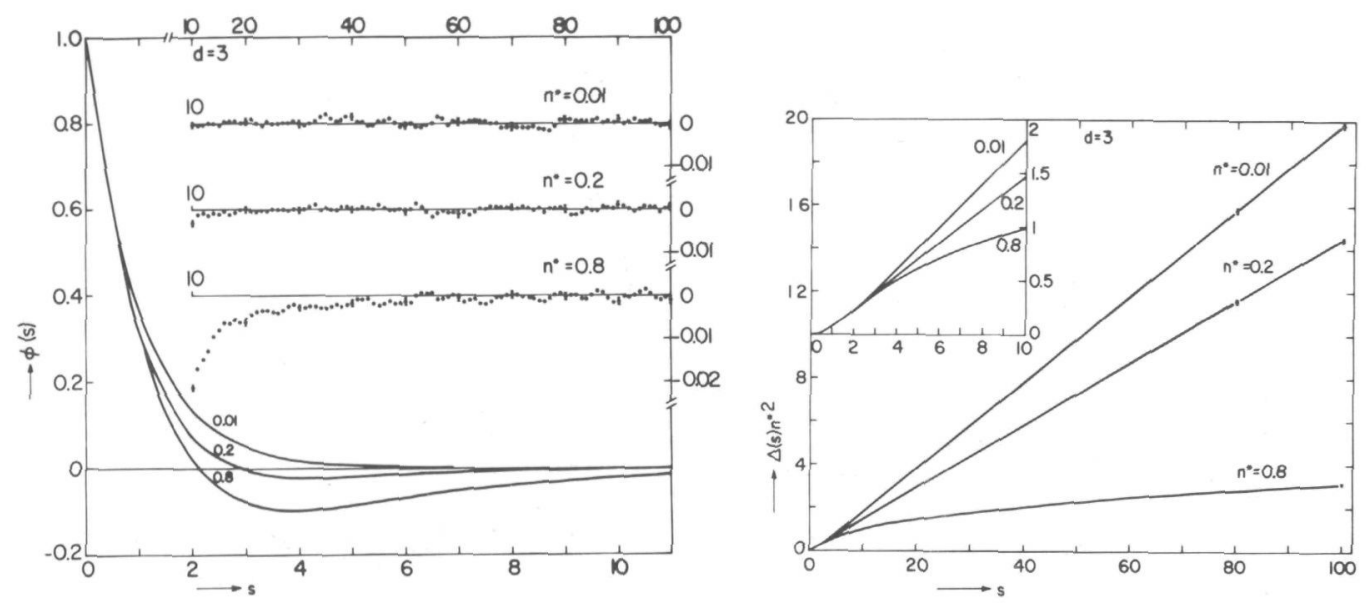

Fig. 4.13. The velocity autocorrelation function $\phi(s)$ for $s<11$ for three densities (lines). The tail for $10<s<100$ is given for the same densities on an entarged scale (dots).

Fig. 4.14. The reduced mean square displacement $\Delta(s) n^{*}$ for three densities.

4.3. Results on the $\mathrm{N}$-dependence

Calculations concerning the dependence of the properties of the Lorentz gas on the number of scatterers $\mathrm{N}$ in the system, still employing periodic boundary conditions, were set up to find values of $\mathrm{N}$ for which the system is indistinguishable, within the limits of the statistical accuracy, from an infinite one. The strategy is to start with $N=1$ and to increase $\mathrm{N}$ until the results are seen to converge. In section 2.4 it has been argued that finite-size effects will be the more serious the lower the density.

In the calculations on the $\mathrm{N}$-dependence the number of collisions performed in each configuration is kept constant (see table 4.5). Therefore, the number of correlation sequences $\mathrm{N}_{f}$ fluctuates somewhat and so does the total number of correlation sequences $N_{f} N_{C}$ used in the averaging 
procedure. For $\mathrm{N}=1$ there is no need to generate configurations with a different position of the scattering centre since the periodic boundary conditions always give a perfect lattice. This lattice breaks up into closed pockets for densities $n^{*}>0.25$ in two dimensions and $n^{*}>\sqrt{2} / 4=0.35$ in three dimensions. The distribution function of the free times $q_{e}\left(s_{k}\right)$

Table 4.5. $N$-dependence in two and three dimensions. In all cases $N_{c}=100$.

\begin{tabular}{|c|c|c|c|c|c|c|}
\hline $\begin{array}{l}1 \\
n^{*}\end{array}$ & $\begin{array}{l}2 \\
\mathrm{~N}\end{array}$ & $\begin{array}{c}3 \\
\mathrm{~N}_{f^{N}}{ }^{\mathrm{C}} \\
\left(10^{5}\right)\end{array}$ & $\tau e^{4 / \tau}$ & $\tau_{N}^{5} / \tau$ & $\begin{array}{l}6 \\
x^{2} \\
(n=1)\end{array}$ & $\begin{array}{c}7 \\
D_{e} / v \sigma \\
\left(s_{x}=50\right)\end{array}$ \\
\hline \multicolumn{7}{|c|}{$d=2$} \\
\hline 0.1 & 1 & 1.0 & $0.688(3)$ & 0.686 & 1959 & $2.26(15)$ \\
\hline 0.1 & 2 & 1.2 & $0.884(24)$ & 0.843 & 315 & $2.62(26)$ \\
\hline 0.1 & 4 & 1.4 & $0.948(21)$ & 0.921 & 75 & $2.10(13)$ \\
\hline 0.1 & 8 & 1.5 & $0.989(17)$ & 0.961 & 22 & $1.94(8)$ \\
\hline 0.1 & 25 & 1.5 & $0.983(10)$ & 0.987 & 3.6 & $1.21(6)$ \\
\hline 0.1 & 50 & 1.5 & $0.995(7)$ & 0.994 & 1.7 & $1.26(5)$ \\
\hline 0.01 & 1 & 1.4 & $0.965(7)$ & 0.969 & 591 & $39(5)$ \\
\hline 0.01 & 2. & 1.5 & $0.992(11)$ & 0.984 & 112 & $37(3)$ \\
\hline 0.01 & 4 & 1.5 & $0.989(9)$ & 0.992 & 63 & $26(2)$ \\
\hline 0.01 & 8 & 1.5 & $0.999(8)$ & 0.996 & 26 & $33(4)$ \\
\hline 0.01 & 25 & 1.5 & $0.999(4)$ & 0.999 & 5.3 & $20(1)$ \\
\hline 0.01 & 50 & 1.5 & $0.993(4)$ & 0.999 & 2.6 & $18(1)$ \\
\hline \multicolumn{7}{|c|}{$d=3$} \\
\hline 0.05 & 1 & 1.2 & $0.797(4)$ & 0.791 & 376 & $2.83(25)$ \\
\hline 0.05 & 2 & 1.3 & $0.911(14)$ & 0.895 & 99 & $3.62(42)$ \\
\hline 0.05 & 4 & 1.4 & $0.964(11)$ & 0.948 & 31 & $2.32(10)$ \\
\hline 0.05 & 8 & 1.5 & $0.979(11)$ & 0.974 & 11 & $2.54(25)$ \\
\hline 0.05 & 25 & 1.5 & $0.987(8)$ & 0.992 & 2.5 & $2.00(7)$ \\
\hline 0.05 & 50 & 1.5 & $0.989(5)$ & 0.996 & 1.5 & $2.03(6)$ \\
\hline
\end{tabular}

is very peculiar for $N=1$. It was found to consist of a number of isolated peaks corresponding to free times between nearest neighbours, next-nearest neighbours and so on in the lattice formed by the periodic boundary condi- 
tions. Beyond a certain time, which varies with the density, the peaks get connected.

For the two-dimensional case an intermediate density $\mathrm{n}^{*}=0.1$ is discussed first. In fig 4.15 the free-times distribution $q_{e}\left(s_{k}\right)$ is shown for some values of $\mathrm{N}$. The significant peaks are easily identified as due to nearest, next-nearest and higher order periodic images of the scatterers. The deviations of the experimental mean free time $\tau_{e}^{/ \tau}$ from 1 are very large but in very good agreement with $\tau_{N} / \tau$ (columns 4 and 5 of table 4.5). The decrease of $x^{2}$, calculated for $q_{e}\left(s_{k}\right)$ with respect to $\hat{Q}\left(s_{k} ; n=1\right)$
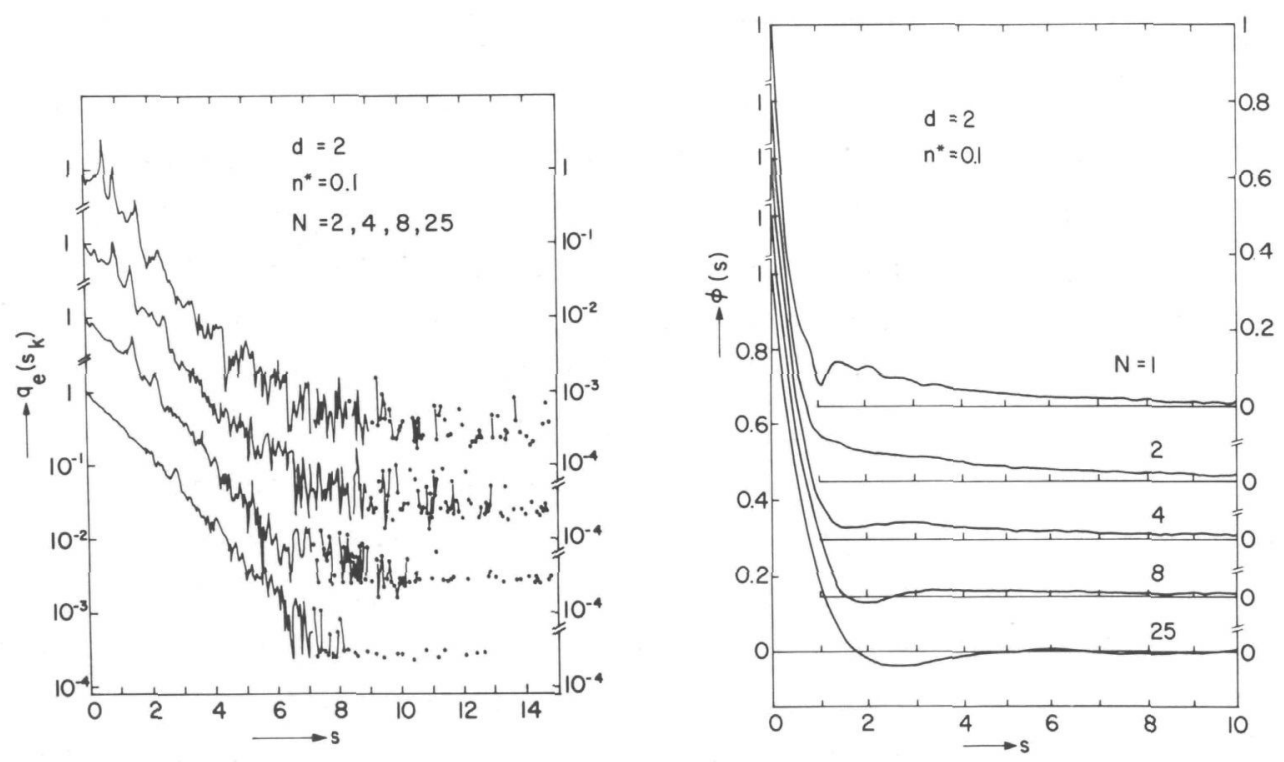

Fig. 4.15. The frequency distribution $q_{e}\left(s_{k}\right)$ for $d=2$ and $n^{*}=0.1$ with increasing $N$ going downwards. Lines have been drawn through the data except for intervals which had zero intensity.

Fig. 4.16. The velocity autocorrelation function $\phi(s)$ for $d=2$ and $n^{*}=0.1$. The positive taiz for smazl $\mathrm{N}$ is clearly demonstrated (although the behaviour for $10<s<50$ is not shown here).

in eq. (4.1), becomes considerable with increasing $N$ (column 6 in table 4.5). 
The diffusion coefficient $D_{e} / v \sigma$, obtained from integration of $\phi(s)$ with a maximum time $s_{x}=50$, is much too large for small $\mathrm{N}$ (last column of table 4.5). It appears that lack of randomness in the system favours diffusion for densities that are not too high. This means that the event of repeated travelling across the system without collisions is dominant. This is also demonstrated in fig. 4.16 by the behaviour of $\phi(s)$. This function decreases relatively slowly for small $\mathrm{N}$ and has a long positive tail which was observed to be significantly different from zero at $s=50$ for $N=1$. For $N=25$ it approaches its asymptotic behaviour, which can also be concluded from the values of $D_{e} / v \sigma$ in table 4.5 and the cases with $\mathrm{N}=500$ and $\mathrm{N}=2000$ for $\mathrm{n}^{*}=0.1$ in table 4.1 .

The other density studied in two dimensions is $n^{*}=0.01$ and this is a rather low one (table 4.5). The limiting value of $\mathrm{D}_{\mathrm{e}} / \mathrm{v} \sigma$ is obtained for larger values of $\mathrm{N}$. The system with $\mathrm{N}=50$ is probably still too small although this cannot be concluded from a calculation with this statistical accuracy. More extensive calculations gave $\mathrm{N}=100$ :

$D_{e} / v \sigma=18.0 \pm 0.2, N=500: D_{e} / v \sigma=17.7 \pm 0.1, N=2000:$

$D_{e} / v \sigma=17.77 \pm 0.03$ and $N=8000: D_{e} / v \sigma=17.80 \pm 0.02$.

From these results it can be concluded that the systems mentioned in table 4.1 are large enough to be free of significant finite-size effects. This is confirmed by the fact that results on the diffusion coefficient for different $N$ at the same density, e.g. for $n^{*}=0.005$ with $\mathrm{N}=2000$ and $\mathrm{N}=8000$, show no significant systematic differences.

In the three-dimensional case the $i$-dependence of one of the lowest densities mentioned in section 4.2, $\mathrm{n}^{*}=0.05$, has been investigated. Here too, $\tau e^{/ \tau}$ is considerably smaller than 1 , but it is in reasonable agreement with $\tau_{N} / \tau$ (table 4.5). For $N=25, D_{e} / v \sigma$ seems to have reached its asymptotic value. So the systems with $N=100$ in table 4.4 are sufficiently large to make finite-size effects neglectable compared to the obtained statistical accuracy. This is also confirmed by the data from the $\mathrm{N}=2000$ systems at the respective densities. However, this is rather surprising in view of the $f$ values calculated in section 2.4. Apparently, travelling once across the system without collisions is less harmful in the 3-dimensional than in the 2-dimensional case. This was also indicated 
by the values of the geometrical factor $\mathrm{f}_{\mathrm{g}}$ given in section 2.4 .

References

4.1) Wood, W.W., private communication

4.2) Alder, B.J. and Wainwright, T.E., Phys. Rev. A1 (1970) 18

4.3) Wood. W.W., (1974) in Fundamental Problems in Statistical Mechanics III Ed. E.G.D. Cohen, North-Holland Publishing Company, Amsterdam 1975 


\section{CONCLUSIONS}

Qualitative evidence has been obtained for a logarithmic term in the density expansion of the inverse diffusion coefficient for the twodimensional Lorentz gas. As the arguments used by Van Leeuwen and Weyland to arrive at the expression for the logarithmic terms are similar to those used for other systems, the evidence found in this case sustains the occurrence of logarithmic terms in other, and more realistic, systems.

The computations are not conclusive with respect to the power-like decay of the velocity autocorrelation function. They do show that the asymptotic behaviour is not dominant before 9 mean free times, even for low densities. This makes it very hard to verify this behaviour.

For the three-dimensional case the results show reasonable agreement for the diffusion coefficient with the predicted density expansion and thus confirm the expectation that evidence for logarithmic terms in three dimensions is very hard to obtain.

As regards the mean free time and distribution of free times good agreement has been observed between theoretical and experimental results, if use is made of a more appropriate alternative average described in this thesis. 
APPENDIX: The joint distribution of the first and second free time for the two-dimensional case.

The derivation given in this appendix is a modification of a calculation performed by Wood ${ }^{2.5)}$ for the overlapping Ehrenfest wind-tree model. Consider a two-dimensional Lorentz gas of $\mathrm{N}$ point-scatterers in a volume $\Omega$, with density $\mathrm{n}=\mathrm{N} / \Omega$, and a moving particle (disk) of radius $\sigma$. Let $t_{1}\left(x, R^{N}\right)$ denote the time until the first collision and $t_{2}\left(x, R^{N}\right)$ the time between the first and the second collision, starting with initial condition $x=(\vec{p}, \vec{q})$ of the moving particle, of which the trajectory in phase space is further completely determined by the configuration of scatterers $R^{N}$. The joint distribution $P_{2}\left(t_{1}, t_{2}\right)$ to be calculated is defined by

$$
\mathrm{P}_{2}\left(t_{1}, t_{2}\right)=\left\langle\delta\left[t_{1}-t_{1}\left(x, R^{N}\right)\right] \delta\left[t_{2}-t_{2}\left(x, R^{N}\right)\right]\right\rangle,
$$

where the average has to be performed over all possible values of $\vec{q}$ and $R^{N}$ and over all possible directions of the velocity $\vec{v}(=\vec{p} / m)$. This average can be reduced without loss of generality to an average over $R^{N}$ alone.

For the joint distribution four factors are important, as will be discussed for the simple case shown in fig. A.1. Here $L_{1}$ is the initial position (of the centre) of the moving particle, L the position at the first collision, $\mathrm{L}_{2}$ the position at the second collision and $\phi$ is the scattering angle for the first collision; the distances $\mathrm{L}_{1} \mathrm{~B}$, $\mathrm{LJ}$ and $\mathrm{L}_{2} \mathrm{E}$ are all equal to $\sigma$ and furthermore one has $L_{1} L=v t_{1}$ and $L L_{2}=v t_{2}$.

1) The probability $f_{1} d t_{1}$ that one of the scatterers gives a first free time between $t_{1}$ and $t_{1}+d t_{1}$ is equal to the product of the density $N /\left(\Omega-\pi \sigma^{2}\right)$ of the scatterers, corrected for the volume of the moving particle, and the area $2 \sigma v d t_{1}$ of width vdt, along CDK. Because of symmetry one needs to consider only the part $C D$, taking a factor 2 into account. So one has $f_{1} d t_{1}=2 \sigma v d t_{1} N /\left(\Omega-\pi \sigma^{2}\right)$. 


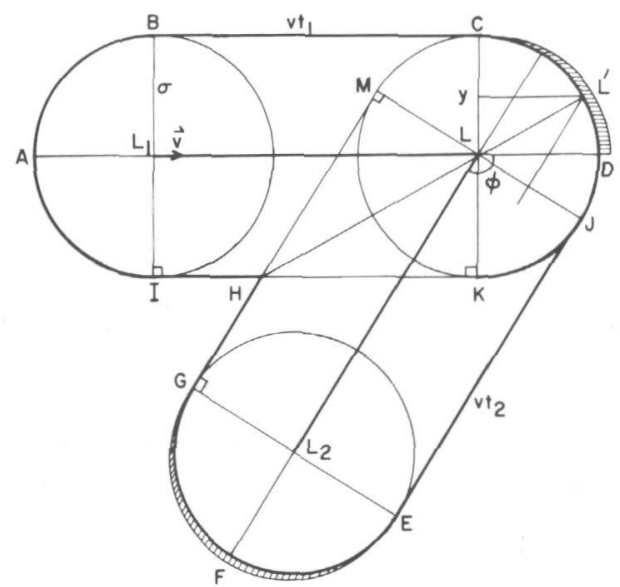

Fig. A.1. Case A with conditions $I K>H K, G M>H M$. $\Omega_{A}=A B C D J E F G H I, \ell_{A}=2, L_{1} B=\sigma, L_{1} L=v t_{1}$ and $L L_{2}=v t_{2}$.

2) The probability $f_{\phi} d \phi$ that the angle between the velocities before and after the first collision is between $\phi$ and $\phi+d \phi$ is proportional to $d y$, with $y$ varying from 0 to $\sigma$ along $D C$. Taking $L^{\prime}$ as the position of the scatterer and requiring that the angle of incidence equals the angle of reflection, one obtains for the relation between $y$ and $\phi$ the result $y=\sigma \cos \frac{1}{2} \phi$. Thus, for $0 \leqslant \phi \leqslant \pi$ one has $f_{\phi} d \phi=\frac{1}{2} \sin \frac{1}{2} \phi d \phi$, where the factor $\sigma$ has been dropped because of normalization.

3) The probability $f_{2} d t_{2}$ that one of the scatterers gives a second free time between $t_{2}$ and $t_{2}+d t_{2}$ is equal to the product of the density $(N-1) /\left(\Omega-\pi \sigma^{2}\right)$ of the remaining scatterers and the area $\ell_{\alpha} \sigma v d t_{2}$ of width vdt 2 along EFG. In the case shown in fig. A.1 one has $\ell_{\alpha}=2$, but in other cases $l_{\alpha}$ may differ from that value; e.g. when for small $t_{2}$ it may occur that $M G<M H$. This results in a factor $f_{2} d t_{2}=l_{\alpha} \sigma v a t_{2}(N-1) /\left(\Omega-\pi \sigma^{2}\right)$.

4) The probability $f_{\Omega}$ that the collisions at time $t_{1}$ and $t_{2}$ do take place is equal to the probability that none of the remaining scatterers lies inside an area $\Omega_{\alpha}$. In fig. A.1. this area is enclosed by the contour ABCDJEFGHI. The expression for $\Omega_{\alpha}$ depends on the case considered. This gives obviously $f_{\Omega}=\left\{\left(\Omega-\Omega_{\alpha}\right) /\left(\Omega-\pi \sigma^{2}\right)\right\}^{N-2}$. Combination of the four 
factors gives for the joint distribution

$$
P_{2}\left(t_{1}, t_{2}\right) d t_{1} d t_{2}=\frac{N(N-1) 2 \sigma^{2} v^{2}}{\left(\Omega-\pi \sigma^{2}\right)^{2}} d t_{1} d t_{2} \int_{0}^{\pi} l_{\alpha}\left\{\frac{\Omega-\Omega \alpha}{\Omega-\pi \sigma^{2}}\right\}^{N-2} \frac{1}{2} \sin \frac{1}{2} \phi d \phi,
$$

which in the limit $\mathrm{N} \rightarrow \infty$ simplifies to

$$
P_{2}\left(t_{1}, t_{2}\right) d t_{1} d t_{2}=2 n^{2} \sigma^{2} v^{2} d t_{1} d t_{2} \int_{0}^{\pi \frac{1}{2} l} \alpha\left(\sin \frac{1}{2} \phi\right)\left\{\exp -n\left(\Omega_{\alpha}-\pi \sigma^{2}\right)\right\} d \phi,
$$

where $l_{\alpha}$ and $\Omega_{\alpha}$ are functions of $t_{1}, t_{2}$ and $\phi$.

For the calculation of $\Omega_{\alpha}$ and $l_{\alpha}$ different cases, as shown in

fig. A.2, have to be considered. The dashed-dotted lines denote boundaries

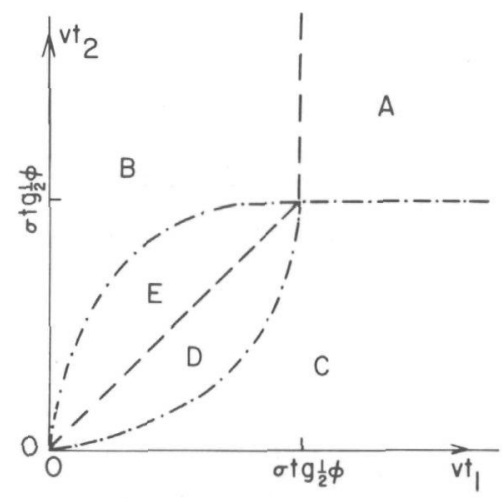

Fig. A.2. Different cases in the calculation of $P_{2}\left(t_{1}, t_{2}\right)$, see text.

on which the expressions of both $\Omega_{\alpha}$ and $l_{\alpha}$ change. On the dashed lines only the expression for $\Omega_{\alpha}$ changes. The envelop of region $E+D$ is drawn for the case $\phi=\pi / 2$, for other values of $\phi$ this region only changes quantitatively. The different cases will now be considered in detail.

Case A. (see fig. A.1). The conditions for this simplest case are 
$v t_{1}>H K$ and $v t_{2}>\mathrm{MH}$, which can be reduced to

$$
v t_{1}>\sigma \operatorname{tg}^{\frac{1}{2}} \phi, \quad v t_{2}>\sigma \operatorname{tg}^{\frac{1}{2}} \phi
$$

The expression for the area $\Omega_{\mathrm{A}}$ is easily obtained by correcting for the overlap of the area's ABCMKI and FGMKJE. The result is

$$
\Omega_{\mathrm{A}}=\pi \sigma^{2}+2 \sigma v t_{1}+2 \sigma v t_{2}-\sigma^{2}\left\{\operatorname{tg}^{\frac{1}{2}} \phi-\frac{1}{2} \phi\right\} .
$$

For $l_{A}$ one has as mentioned above

$$
\ell_{A}=2 .
$$

In fig. A.1 a situation for $\phi>\frac{1}{2} \pi$ has been drawn, for $\phi<\frac{1}{2} \pi$ one obtains in a similar way the same expressions for $\Omega_{A}$ and $\ell_{A}$. This will hold true for all cases to be discussed.

Case B. (see fig. A.3). The conditions for this case are $v t_{1}<H K$ and $v t_{2}>M S$. It is obvious that the condition on $v t_{2}$ can be taken to

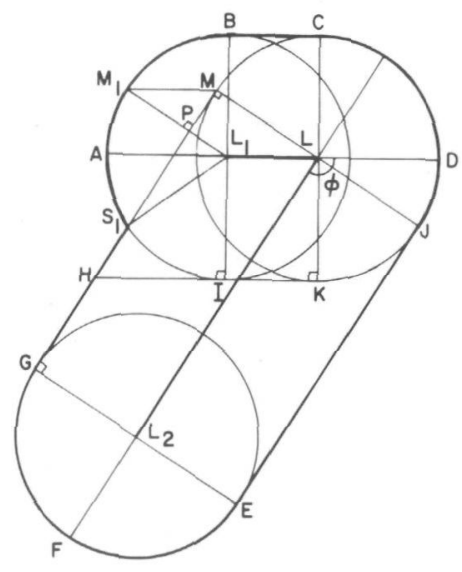

Fig. A.3. Case B with conditions $I K<H K, G M>S_{1} M$. $\Omega_{B}=A B C D J E F G S_{1}, l_{B}=2, L_{1} B=\sigma, L_{1} L=v t_{1}$ and $L L_{2}=v t_{2}$.

be weaker than $\mathrm{vt}_{2}>\mathrm{MH}$, what one might expect from case $\mathrm{A}$. It is convenient for the evaluation of $\Omega_{B}$ to choose a point $M_{1}$ such that 
$\mathrm{MM}_{1} / / \mathrm{CB}$; then one also has $\mathrm{M}_{1} \mathrm{~L}_{1} / / \mathrm{ML}$. By evaluating now the areas $M_{1} M C B, \quad M_{1} M P$ and $M_{1} P_{1} A$ one obtains

$$
\begin{aligned}
& \Omega_{B}=\pi \sigma^{2}+2 \sigma v t_{2}+\sigma v t_{1}(1+\cos \phi)-\frac{1}{2}\left(v t_{1}\right)^{2} \sin \phi \cos \phi \\
& +\frac{1}{2} \sigma^{2} \arccos \left[1-\left(v t_{1} / \sigma\right) \sin \phi\right]-\frac{1}{2}\left(\sigma-v t_{1} \sin \phi\right)\left[2 \sigma v t_{1} \sin \phi-\right. \\
& \left.-\left(v t_{1} \sin \phi\right)^{2}\right]^{\frac{1}{2}} .
\end{aligned}
$$

It is obvious that one has again

$$
e_{B}=2 \text {. }
$$

The explicit expression for the conditions on case $B$ reads

$$
\begin{aligned}
& v t_{1}<\sigma \operatorname{tg}^{\frac{1}{2} \phi}, \quad v t_{2}>\left[2 \sigma v t_{1} \sin \phi-\left(v t_{1} \sin \phi\right)^{2}\right]^{\frac{1}{2}}-v t_{1} \cos \phi \cdot \quad \text { (A.9) } \\
& \text { Case C. (see fig. A.4). The conditions for this case are } v t_{1}>\mathrm{KS}_{2}
\end{aligned}
$$
and $\mathrm{vt}_{2}<\mathrm{HM}$. This case is identical to case B after interchanging the

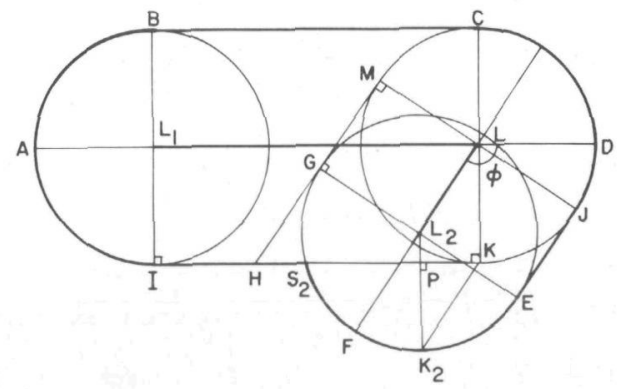

Fig. A.4. Case C with conditions $G M<H M$, IK $>S_{2} K$.

$$
\Omega_{C}=A B C D J E F S_{2} I,{ }_{C}=\text { projection of are } S_{2} E \text { onto } G E, L_{1} B=\sigma \text {, }
$$
$L_{1} L=v t_{1}$ and $L L_{2}=v t_{2}$.

variables $t_{1}$ and $t_{2}$, except for the expresssion for $\ell_{C}$. Here a point $k_{2}$ is chosen, $\mathrm{KK}_{2} / / \mathrm{JE}$. By calculating now the areas $\mathrm{K}_{2} \mathrm{KJE}, \mathrm{K}_{2} \mathrm{KP}$ and 
$\mathrm{K}_{2} \mathrm{PS}_{2} \mathrm{~F}$ one finds

$$
\begin{aligned}
& \Omega_{C}=\pi \sigma^{2}+2 \sigma v t_{1}+\sigma v t_{2}(1+\cos \phi)-\frac{1}{2}\left(v t_{2}\right)^{2} \sin \phi \cos \phi \quad \text { (A. 10) } \\
& +\frac{1}{2} \sigma^{2} \arccos \left[1-\left(v t_{2} / \sigma\right) \cos \phi\right]-\frac{1}{2}\left(\sigma-v t_{2} \sin \phi\right)\left[2 \sigma v t_{2} \sin \phi-\left(v t_{2} \sin \phi\right)^{2}\right]^{\frac{1}{2}} .
\end{aligned}
$$

Because the arc $\mathrm{GS}_{2}$ lies inside the region where no scatterers are allowed, the value of $l_{C}$ differs from two:

$$
l_{C}=1+\left[1-\left(v t_{2} / \sigma\right) \sin \phi\right] \cos \phi+\left[2\left(v t_{2} / \sigma\right) \sin \phi-\left(v t_{2} / \sigma\right)^{2} \sin ^{2} \phi\right]^{\frac{1}{2}} \sin \phi .
$$

The conditions on case $\mathrm{C}$ read in detail

$$
v t_{1}>\left[2 \sigma v t_{2} \sin \phi-\left(v t_{2} \sin \phi\right)^{2}\right]^{\frac{1}{2}}-v t_{2} \cos \phi, v t_{2}<\sigma \operatorname{tg} \frac{1}{2} \phi .
$$

Case D. (see fig. A.5). The remaining region (see fig. A.2) is dealt with by considering, for convenience of the analysis as well as the numerical treatment, two more cases $\mathrm{D}$ and $\mathrm{E}$. The whole region could be covered by any one of thesecases. The boundary between the two corresponding areas is thus arbitrary and the line $v t_{1}=v t_{2}$ has been chosen because of symmetry. For case D obviously the following conditions hold:

$$
v t_{2}<v t_{1}<\left[2 \sigma v t_{2} \sin \phi-\left(v t_{2} \sin \phi\right)^{2}\right]^{\frac{1}{2}}-v t_{2} \cos \phi .
$$

The calculation of $\Omega_{D}$ is similar to that of $\Omega_{C^{\prime}}$ except for the areas $\mathrm{K}_{2}$ PIU and UIS which lead to rather complicated expressions. The distance $\mathrm{L}_{1} \mathrm{~L}_{2}$ will be denoted by

$$
e_{12}=\left[\left(v t_{1}\right)^{2}+\left(v t_{2}\right)^{2}+2 v t_{1} v t_{2} \cos \phi\right]^{\frac{1}{2}} .
$$

One finds then

$$
\Omega_{D}=\pi \sigma^{2}+2 \sigma v t_{1}+\sigma v t_{2}(1+\cos \phi)-\frac{1}{2}\left(v t_{2}\right)^{2} \sin \phi \cos \phi+
$$




$$
\begin{aligned}
& +\frac{1}{2} \sigma^{2} \zeta-\frac{1}{2}\left(\sigma-v t_{2} \sin \phi\right)\left(v t_{1}+v t_{2} \cos \phi\right)+\frac{1}{2} \sigma^{2}(\nu-u) \\
& -\frac{1}{2}\left(\sigma^{2}-2 \sigma v t_{2} \sin \phi+l_{12}^{2}\right)^{\frac{1}{2}} l_{12} \sin \rho \sin \nu / \sin (\rho+\xi),
\end{aligned}
$$

where a number of auxiliary angles have been introduced (see also fig. A.5):

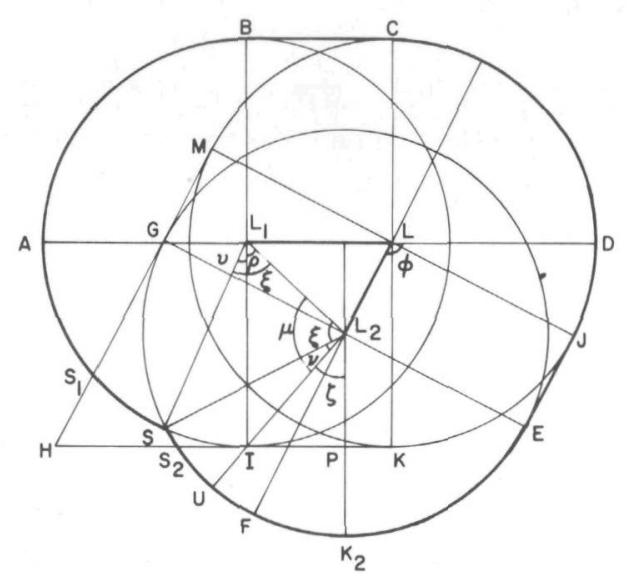

Fig. A.5. Case D with conditions $I K<S_{2} K$, IK > GM.

$\Omega_{D}=A B C D J E F S, l_{D}=$ projection of are $S E$ onto $G E, L_{1} B=\sigma$, $L_{1} L=v t_{1}$ and $L L_{2}=v t_{2}$.

$$
\begin{aligned}
& \zeta=L I L_{2} P=\operatorname{arctg}\left(v t_{1}+v t_{2} \cos \phi\right) /\left(\sigma-v t_{2} \sin \phi\right), \\
& v=\mu-\xi, \\
& v=\xi-\rho,
\end{aligned}
$$

$\mu=L I L_{2} L_{1}=\arccos \left(l_{12}^{2}-\sigma v t_{2} \sin \phi\right) / l_{12}\left(\sigma^{2}-2 \sigma v t_{2} \sin \phi+l_{12}^{2}\right)^{\frac{1}{2}}$,

$\xi=L_{1} L_{2} L_{1}=\arccos \ell_{12} / 2 \sigma$, 


$$
\rho=L I L_{1} L_{2}=\arccos v t_{2} \sin \phi / l_{12} .
$$

By evaluating the arc GS in this case, one finds for the set of allowed positions of scatterers along arc SE:

$$
l_{D}=1+\left(v t_{1} / 2 \sigma\right) \sin \phi+\left(v t_{1} \cos \phi+v t_{2}\right)\left\{l_{12}^{-2}-\left(4 \sigma^{2}\right)^{-1}\right\}^{\frac{1}{2}}
$$

Case $E$. This case needs not to be discussed in detail. $\Omega_{E}$ can be obtained from $\Omega_{D}$ by interchanging $v t_{1}$ and $v t_{2}$. The expression for $l_{E}$ is identical to the one for $l_{D}$. The conditions for this case read

$$
v t_{1}<v t_{2}<\left[2 \sigma v t_{1} \sin \phi-\left(v t_{1} \sin \phi\right)^{2}\right]^{\frac{1}{2}}-v t_{1} \cos \phi .
$$

As a check on the expressions for the different cases it can be shown that they match on the boundaries. It is appropriate to reduce time with respect to the Boltzmann mean free time, as has been done in chapter 2 . We define

$$
s_{1}=2 n \sigma v t_{1}=t_{1} / \tau, \quad s_{2}=2 n \sigma v t_{2}=t_{2} / \tau
$$

and moreover a reduced volume $\Omega_{\alpha}^{*}$ given by

$$
\Omega_{\alpha}^{*}=\mathrm{n}\left(\Omega_{\alpha}-\pi \sigma^{2}\right)
$$

Substituting (A.19) and (A.20) into (A.3) and taking into account the Jacobian of the transformation, one finds for the reduced joint distribution

$$
\mathrm{P}_{2}^{*}\left(\mathrm{~s}_{1}, \mathrm{~s}_{2}\right) \mathrm{ds}_{1} \mathrm{ds}_{2}=\mathrm{ds} \mathrm{ds}_{2} \int_{0}^{\pi} \mathrm{d \phi} \frac{1}{4} l_{\alpha} \sin \frac{1}{2} \phi \exp -\Omega_{\alpha}^{*} .
$$

It should be noted that depending upon the pertaining values of $\mathrm{s}_{1}, \mathrm{~s}_{2}$, 
$\phi$ and $n^{*}$ the correct expressions for $\Omega_{\alpha}^{*}$ and $l_{\alpha}$ have to be chosen from cases $A$ until E.

The joint distribution $\mathrm{P}_{2}^{*}\left(\mathrm{~s}_{1}, \mathrm{~s}_{2}\right)$ can be obtained from (A.21) by numerical integration over $\phi$. The distribution of the first free time $\mathrm{P}_{2,1}^{*}\left(\mathrm{~s}_{1}\right)$ can be obtained subsequently by numerical integration over $s_{2}$. This serves as a check on the whole procedure since this result should be identical to the result for $\mathrm{P}(\mathrm{t})$, see $(2.54)$. Numerical integration of $\mathrm{P}_{2}^{*}\left(\mathrm{~s}_{1}, \mathrm{~s}_{2}\right)$ over $s_{1}$ gives the distribution of the second free time $\mathrm{P}_{2,2}^{*}\left(\mathrm{~s}_{2}\right)$. Subsequent integration over $s_{2}$ gives another check, since the result should be equal to one because of normalization. For a number of densities the distribution of the second free time $\mathrm{P}_{2,2}^{*}\left(\mathrm{~s}_{2}\right)$ is shown in fig. A.6, together with a pure exponential. The deviation form a pure exponential increases as a function of the density. For $\mathrm{s}_{2}=0$ the distribution is independent of the density, in fact $P_{2,2}^{*}(0)$ can be evaluated exactly to give $1 / 3$. In table A.1 the first moment $\left\langle\mathrm{s}_{2}\right\rangle$ of the distribution of the second free time $\mathrm{P}_{2}^{*}, 2\left(\mathrm{~s}_{2}\right)$ is given as a function of the density. The deviation of the

Table A.1. The average of the second free time $\left\langle s_{2}\right\rangle$ as a function of the density (the time $s_{2}$ is reduced with respect to the Boltzmann mean free time $\tau$ ).

\begin{tabular}{|l|l|}
\hline$n^{*}$ & $\left.<\mathrm{s}_{2}\right\rangle$ \\
\hline 0.005 & 1.02 \\
0.01 & 1.03 \\
0.02 & 1.05 \\
0.05 & 1.10 \\
0.1 & 1.14 \\
0.2 & 1.21 \\
0.5 & 1.31 \\
1.0 & 1.37 \\
10. & 1.43 \\
\hline
\end{tabular}

average of the second free time from the Boltzmann mean free time increases with density.

At the densities for which the numerical integration has been performed, the interval 0 to $\pi$ for $\phi$ has been divided into 40 intervals. 


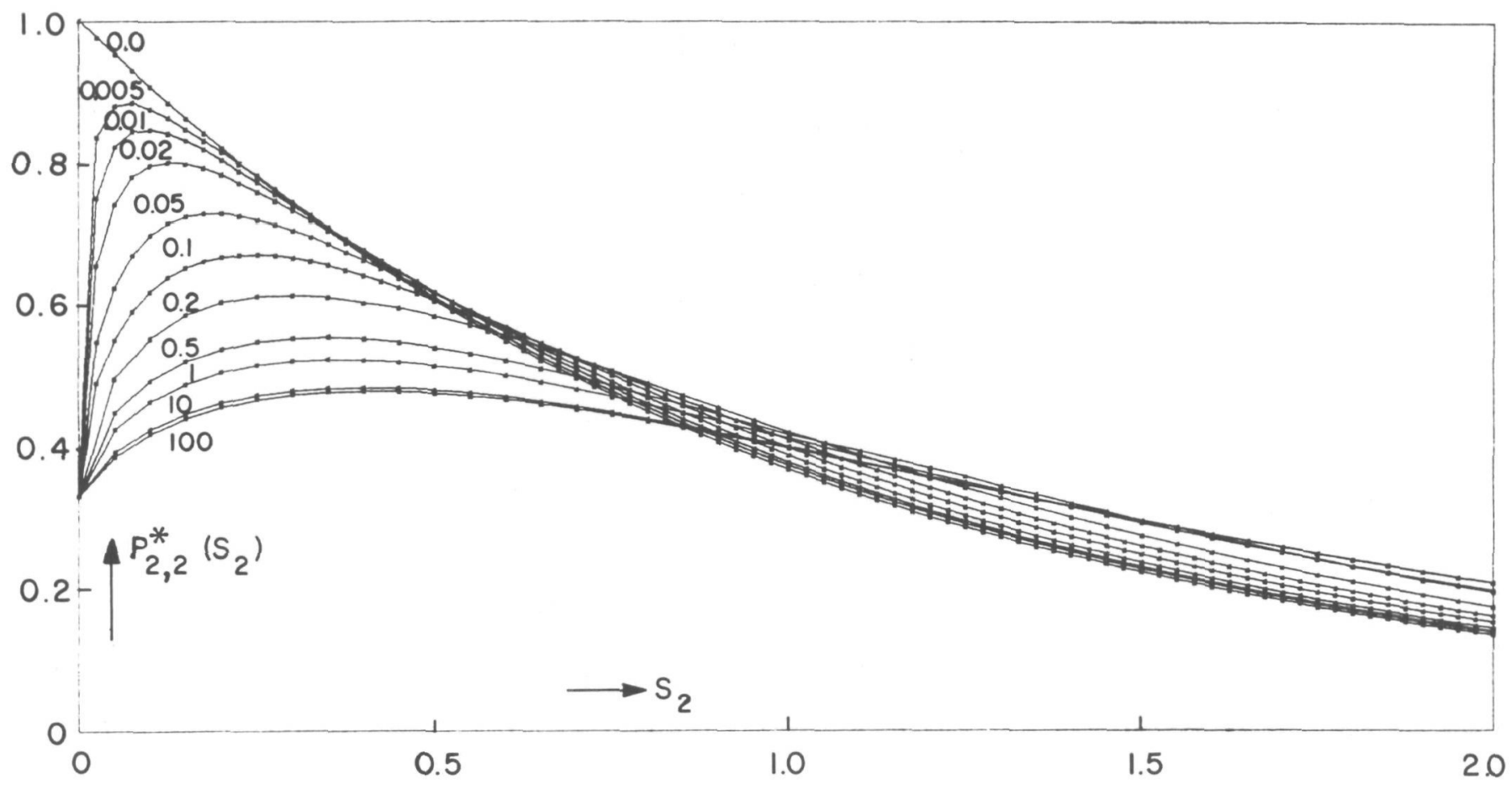

Fig. A.6. The distribution of the second free time $P_{2,2}^{*}\left(s_{2}\right)$ for a number of densities. For the density $n^{*}=0$ the distribution is a pure exponential. 
The maximum value for $\mathrm{s}_{1}$ and $\mathrm{s}_{2}$ used was 7.5 ; this range has been divided into 150 intervals for densities $n^{*}>0.1$ and into 300 intervals for the lower ones. For all integrations the Simpson (3-point) rule has been used, giving an accuracy of better than $1 \%$. 


\section{SUMMARY}

The numerical study of the Lorentz gas has been undertaken as to obtain quasi-experimental data on the density dependence of the diffusion coefficient. Primarily the 2-dimensional case has been studied, for which the deviations from a pure power series in the density for the inverse diffusion coefficient are expected to be most dominant. In the 1-dimensional case there is no diffusion at all and for 3 dimensions the expected deviations are relatively small.

For a more substantial comparison of theory and experiment, two additional terms in the series expansion of the inverse diffusion coefficient have been calculated for the 2-dimensional case: the regular term of the same order in density as the dominant logarithmic term and the most important logarithmic term of one order higher in the density.

As a check on the computations, especially for the Monte-Carlo part of it, expressions have been derived for the distribution function of free times and for the mean free time.

For the computation of the static quantities (initial conditions) the crude Monte-Carlo method has been used. The time-dependent behaviour has been computed with the method of molecular dynamics (solving the equations of motion). For the Monte-Carlo method the generator of pseudo-random numbers is essential and its properties have been studied. In this respect the distribution function of free times appeared to be useful. Optimalization of the molecular-dynamics computations, especially dividing the system into sub-systems (cells), resulted in a considerable saving of computing time.

The computed diffusion coefficients for the 2-dimensional case at low densities are in good agreement with the predicted dominant logarithmic term. The contribution of the two terms calculated in addition to the ones already known is not quite satisfactory. 
The dependence of the results on the size of the system has been investigated experimentally. However this investigation could be facilitated by using an expression describing the density dependence of finitesize effects. The influence of finite $\mathrm{N}$ on the cases reported is smaller than the statistical accuracy obtained. 
SAMENVATTING

De numerieke studie van het lorentzgas is ondernomen teneinde quasiexperimentele gegevens te verkrijgen omtrent de dichtheidsafhankelijkheid van de diffusiecoefficient. De aandacht is hierbij vooral gericht op het 2-dimensionale geval waar de verwachte afwijkingen van een zuivere machtreeks in de dichtheid voor de reciproke diffusiecoefficient het grootst zijn. In het 1 -dimensionale geval is er geen diffusie en voor 3 dimensies zijn de verwachte afwijkingen gering.

Voor een meer solide vergelijking tussen theorie en experiment zijn twee extra termen in de reeksontwikkeling van de reciproke diffusiecoefficient berekend voor het 2-dimensionale geval: de reguliere term van dezelfde orde in de dichtheid als de dominante logarithmische term en de belangrijkste logarithmische term van één orde hoger in de dichtheid.

Als toetsen voor de numerieke berekeningen, en vooral voor het monte-carlo aspect ervan, zijn uitdrukkingen afgeleid voor de verdelingsfunctie van de vrije weglengten en voor de gemiddelde vrije weglengte.

Voor de uitvoering van de numerieke berekeningen is voor de statischegrootheden de eenvoudige monte-carlomethode gebruikt en voor het tijdafhankelijk gedrag de methode van moleculaire dynamica. Voor de monte-carlomethode is aan de generator van pseudo-randomgetallen de nodige aandacht besteed. De verdelingsfunctie van vrije weglengten blijkt in dit opzicht een nuttig hulpmiddel. Optimalisering van de moleculairedynamicaberekeningen, vooral de verdeling van het systeem in cellen, leidt tot een grote besparing van rekentijd.

De berekende diffusiecoefficienten voor het 2-dimensionale geval bij lage dichtheden zijn in goede overeenstemming met de voorspelde dominante logaritrmische term. De bijdrage van de twee extra berekende termen voldoet niet aan de verwachtingen.

De afhankelijkheid van de resultaten van de grootte van het systeem 
is voornamelijk experimenteel onderzocht, $z i j$ het met behulp van een theoretische voorspelling voor de afhankelijkheid van de dichtheid. De invloed van eindige $N$ voor de beschouwde gevallen is kleiner dan de bereikte statistische nauwkeurigheid. 
ACKNOWLEDGEMENTS

I thank Dr. A. Compagner for the suggestion to start computer experiments and for many discussions and professor J.J. van Loef for giving me the opportunity to work in his group.

I thank professor J.M.J. van Leeuwen for raising the subject, for his continuous interest and for analyzing the diagrams. I also thank

dr. A. Weijland, dr. M.H. Ernst and dr. W.W. Wood for helpful discussions.

I am much indebted to Mr. J.C. van Rijs for his help in preparing computer programs, to Miss M. Ritsema for type-writing this thesis and to Mrs. N. Halbe for making the drawings.

The large amount of machine time so generously granted by the computing Centre of the Delft University of Technology and the attentive cooperation of the operators night and day are gratefully acknowledged. 

STELLINGEN

I

Als controle op de correcties van resultaten van neutronenverstrooiingsexperimenten is het aan te bevelen moleculaire-dynamicaberekeningen te doen.

C. Bruin en A. Hasman, Phys. Letters 33A (1970) 338

A. Hasman en P. Zandveld, Phys. Letters 34A (1971) 112

II

De bewering dat simulaties in de fysica niet voorkomen is aanvechtbaar. C.A. van Peursen en S. Boendermaker, Intermediaix 12 e jaargang $\underline{3}$ (1976) 25

III

Het model voor de sueltovergang van Briant en Burton heeft voor elke temperatuur een 'ergodische potentiaal' in de faseruimte als men met behulp van een markovproces middelt over het kanoniek ensemble.

Dit vermindert de relevantie van dit model voor de door hen onderzochte smeltovergang van microclusters, waar voor lage temperaturen de faseruimte niet ergodisch blijkt te zijn voor middelingen over het kanoniek ensemble berekend met behulp van een markovproces.

C.L. Briant en J.J. Burton, J. Chem. Phys. 63 (1975) 2045 
De introductie van een potentiaalbarrière met ruimtelijke uitgebreidheid in het model voor de smeltovergang van Briant en Burton zal leiden tot realistischer resultaten voor gemiddelden over het kanoniek ensemble. C.L. Briant en J.J. Burton, J. Chem. Phys. 63 (1975) 2045

\section{$\mathrm{V}$}

Het kan bewezen worden dat in een 1-dimensionaal roostergas bij constante druk de dichtheid kleiner is naarmate de roosterconstante kleiner is. Dezelfde relatie tussen dichtheid en roosterconstante geldt op de bodem van een lineaire atmosfeer.

C. Bruin en A. Compagner, Physica 68 (1973) 171

VI

Wanneer de coefficient van zelfdiffusie op de juiste wijze met behulp van de enskogtheorie berekend wordt, is er uitstekende overeenkomst met zowel de experimentele waarde als die uit het computerexperiment. C. Bruin, Phys. Letters 28A (1969) 778

VII

Voor de bewering van Cicotti en Jacucci dat hun methode voor simulatie van de dynamische responsie van een systeem niet geschikt is voor effecten op tamelijk lange tijden is hun argument voor het exponentiëel uiteengegaan van trajectoriën in de faseruimte niet voldoende. De invloed van de grootte van het uitwendige veld zal daartoe eerst onderzocht moeten worden.

G. Cicotti en G. Jacucci, Phys. Rev. Letters 35 (1975) 789

S.D. Stoddard en J. Ford, Phys. Rev. A8 (1973) 1504 
Gezien het belang dat de afdeling Technische Natuurkunde hecht aan fundamenteel onderzoek als onderdeel in de opleiding tot natuurkundig ingenieur verdienen fysisch georienteerde computersimulaties in deze opleiding een plaats.

IX

Programma's die terwille van de statistische nauwkeurigheid veel computertijd vergen zouden, bij onvoldoende ruimte in de nachten gedurende de werkweek, in het weekeinde gedraaid moeten kunnen worden. Berekeningen van dit type vermeld in dit proefschrift hadden ruimschoots uitgevoerd kunien worden door de computer gedurende een half jaar tijdens de weekeinden niet uit te schakelen. 